\title{
Constraining the structure and formation of the Galactic bulge from a field in its outskirts $\star, \star \star$
}

\section{FLAMES-GIRAFFE spectra of about 400 red giants around $(I, b)=\left(0^{\circ},-10^{\circ}\right)$}

\author{
S. Uttenthaler ${ }^{1}$, M. Schultheis ${ }^{2,3}$, D. M. Nataf ${ }^{4}$, A. C. Robin ${ }^{2}$, T. Lebzelter ${ }^{1}$, and B. Chen ${ }^{2}$ \\ 1 University of Vienna, Department of Astronomy, Türkenschanzstraße 17, 1180 Vienna, Austria \\ e-mail: [stefan.uttenthaler; thomas.lebzelter] @univie.ac.at \\ 2 Institut Utinam, CNRS UMR6213, OSU THETA, Université de Franche-Comté, 41bis avenue de l'Observatoire, 25000 Besançon, \\ France \\ e-mail: [mathias; annie.robin]@obs-besancon. fr \\ 3 Institut d'Astrophysique de Paris, UMR 7095 CNRS, Université Pierre et Marie Curie, 98bis boulevard Arago, 75014 Paris, France \\ ${ }^{4}$ Department of Astronomy, Ohio State University, 140 West 18th Avenue, Columbus, OH 43210, USA \\ e-mail: nataf@astronomy.ohio-state.edu
}

Received 16 February 2012 / Accepted 30 July 2012

\begin{abstract}
Context. The presence of two stellar populations in the Milky Way bulge has been reported recently, based on observations of giant and dwarf stars in the inner and intermediate bulge.

Aims. We aim at studying the abundances and kinematics of stars in the outer Galactic bulge, thereby providing additional constraints on formation models of the bulge.

Methods. Spectra of 401 red giant stars in a field at $(l, b)=\left(0^{\circ},-10^{\circ}\right)$ were obtained with the FLAMES-GIRAFFE spectrograph at the VLT. Stars of luminosities down to below the two bulge red clumps are included in the data set. From these spectra we measured general metallicities, abundances of iron and the $\alpha$-elements, and radial velocities of the stars. The abundances were derived from an interpolation and fitting procedure within a grid of COMARCS model atmospheres and spectra. These measurements as well as photometric data were compared to simulations with the Besançon and TRILEGAL models of the Galaxy.

Results. We confirm the presence of two populations among our sample stars: i) a metal-rich one at $[\mathrm{M} / \mathrm{H}] \sim+0.3$, comprising about $30 \%$ of the sample, with low velocity dispersion and low $\alpha$-abundance, and ii) a metal-poor population at $[\mathrm{M} / \mathrm{H}] \sim-0.6$ with high velocity dispersion and high $\alpha$-abundance. The metallicity difference between the two populations, a systematically and statistically robust figure, is $\Delta[\mathrm{M} / \mathrm{H}]=0.87 \pm 0.03$. The metal-rich population could be connected to the Galactic bar. We identify this population as the carrier of the double red clump feature. We do not find a significant difference in metallicity or radial velocity between the two red clumps, a small difference in metallicity being probably due to a selection effect and contamination by the metal-poor population. The velocity dispersion agrees well with predictions of the Besançon Galaxy model, but the metallicity of the "thick bulge" model component should be shifted to lower metallicity by 0.2 to 0.3 dex to well reproduce the observations. We present evidence that the metallicity distribution function depends on the evolutionary state of the sample stars, suggesting that enhanced mass loss preferentially removes metal-rich stars. We also confirm the decrease of $\alpha$-element over-abundance with increasing metallicity. Conclusions. Our sample is consistent with the existence of two populations, one being a metal-rich bar, the second one being more like a metal-poor classical bulge with larger velocity dispersion.
\end{abstract}

Key words. Galaxy: bulge - Galaxy: formation - Galaxy: kinematics and dynamics - stars: abundances - stars: late-type

\section{Introduction}

The bulge of the Milky Way galaxy is such a complex system that its formation and evolution is still poorly understood. There exist two main scenarios for the Galactic bulge (GB) formation. The first one, called the "classical" scenario, describes the bulge formation through initial collapse of gas at early times (Eggen et al. 1962) or through hierarchical merging of sub-clumps (Noguchi 1999; Aguerri et al. 2001). In the monolithic collapse

\footnotetext{
* Based on observations at the Very Large Telescope of the European Southern Observatory, Cerro Paranal/Chile under Programme 083.D-0046(A).

$\star \star$ Table 3 is available only at the CDS via anonymous ftp to cdsarc.u-strasbg.fr $(130.79 .128 .5)$ or via http://cdsarc.u-strasbg.fr/viz-bin/qcat?]/A+A/546/A57
}

case the bulge formed before the disc and the star-formation time-scale was very short $(\sim 0.5 \mathrm{Gyr}$; Thomas et al. 2005$)$. The resulting stars are old ( $\gtrsim 10 \mathrm{Gyr})$ and have enhancements of $\alpha$-elements relative to iron in a large range of $\mathrm{Fe}$ abundances, which are characteristic of classical bulges. This indicates a very fast bulge formation, where SNe Ia did not have time to pollute the gas with $\alpha$-element-free ejecta. In the hierarchical merging the bulge also formed before the disc but on a longer timescale (of the order of a few Gyr; Noguchi 1999) and therefore this approach predicts lower over-abundances of $\alpha$-elements.

The second scenario, called the "pseudo-bulge" scenario, describes the bulge formation by the buckling of the disc, following a disc instability (also called a bar; Combes et al. 1990; Raha et al. 1991; Norman et al. 1996; Kormendy \& Kennicutt 2004; Athanassoula 2005). In this case the bulge forms after the disc 
and on a much longer timescale than a classical bulge. After the bar formation the disc is heated in vertical direction (Combes \& Sanders 1981), giving rise to the typical boxy/peanut shape. The bulge formed in this way will be a mixture of disc stars and stars formed in situ by gas which is likely to be well mixed by the action of the bar. In this scenario, the enhancement of the $\alpha$-elements in the bulge stars is predicted to be low, similar to that of the inner disc stars in the models.

It should be noted that, in addition to the classical bulge and pseudo-bulge classifications, an alternative formation mechanism has been suggested, namely the "clump-origin bulge". In this scenario, first proposed by Noguchi (1998) and recently elaborated upon by Inoue \& Saitoh (2011), stellar clumps spontaneously form in high gas-density discy galaxies, as shown by $N$-body/SPH simulations, and dynamical friction drags these clumps to the centre of the galaxy where they aggregate into a bulge-like structure. The models of Inoue \& Saitoh (2011) reproduce the observed boxy shape, rapid star formation, and vertical metallicity gradients observed in the bulge of the Milky Way. Clumpy galaxies that could be the analogues of the primordial Milky Way and that are consistent with these models have been observed in the high-redshift universe (Elmegreen \& Elmegreen 2005; Genzel et al. 2011), but also locally (Elmegreen et al. 2012).

Each of these scenarios is supported by at least some of the observational constraints. The question of formation history is crucial and necessary to investigate because our Galaxy is a benchmark for understanding the formation of disc galaxies. Recently, Babusiaux et al. (2010) and Hill et al. (2011) analysed large samples of red clump stars in Baade's window and three fields close to the minor axis, at $b \sim-4^{\circ},-6^{\circ}$, and $-12^{\circ}$, which revealed the presence of two distinct populations: a metal-poor component around $[\mathrm{Fe} / \mathrm{H}] \approx-0.3$ dex with a broad distribution in $[\mathrm{Fe} / \mathrm{H}]$, and metal-rich component centred around $[\mathrm{Fe} / \mathrm{H}] \approx$ +0.3 with a small spread in metallicity. In addition, these two populations show kinematical differences: the metal-poor component is compatible with an old spheroid, whereas the metalrich component is consistent with a bar population. Therefore, two different formation scenarios have been proposed: a rapid formation timescale for the metal-poor component, and a formation over a longer timescale driven by the evolution of the bar (pseudo-bulge) for the metal-rich component. Similarly, Bensby et al. (2011) find two populations in their sample of micro-lensed bulge dwarf and sub-giant stars, centred at $[\mathrm{Fe} / \mathrm{H}] \approx-0.6$ and $[\mathrm{Fe} / \mathrm{H}] \approx+0.3$, with a dearth of stars around $[\mathrm{Fe} / \mathrm{H}]=0.0$.

Other observations favoured one or the other scenario, which prevented a clear picture of bulge formation to emerge. For example, Zoccali et al. (2008), from the observed sample reanalysed later by Babusiaux et al. (2010), found a clear metallicity gradient in the bulge; this was interpreted as a challenge to the scenario in which the bulge would result solely from the vertical heating of the bar. On the other hand, recent radial velocity studies (Howard et al. 2008, 2009; Shen et al. 2010; Kunder et al. 2012) find that the radial velocity dispersion $\left(\sigma_{\mathrm{RV}}\right)$ of bright bulge giants is fully consistent with the pseudo-bulge scenario. In particular, Shen et al. (2010) show that any classical bulge contribution cannot be larger than $\sim 8 \%$ of the disc mass. Furthermore, recent spectroscopic studies of the abundances of the $\alpha$-elements as a function of $[\mathrm{Fe} / \mathrm{H}]$ revealed that at least the metal-poor bulge stars are chemically similar to stars in the local thick disc (Meléndez et al. 2008; Alves-Brito et al. 2010; Ryde et al. 2010) and the inner disc at Galactocentric distances of 4 to $7 \mathrm{kpc}$ (Bensby et al. 2010b).
A comprehensive summary of the current observational picture of the bulge structure, formation, and evolution cannot be given in this paper. Instead, we refer to the recent review by Rich (2011). In any case, it is clear that the current observational evidence is not sufficient to conclusively constrain the structure and formation history of the Galactic bulge, and that more observations are required.

In the past, most of the attention has been paid to the intermediate bulge, e.g. Baade's window towards $(l, b)=\left(+1^{\circ},-3.9^{\circ}\right)$, while the outer bulge has been somewhat neglected. This may explain why an important feature such as the double red clump (RC), which becomes apparent only at $|b| \gtrsim 5^{\circ}$, was detected only recently (Nataf et al. 2010; McWilliam \& Zoccali 2010). This feature is interpreted as two over-densities of bulge stars at different distances from the sun (McWilliam \& Zoccali 2010; Saito et al. 2011). It is suggested that the over-densities look like a three-dimensional X-structure (McWilliam \& Zoccali 2010). A similar X-shaped structure has been confirmed to also exist in bar/boxy bulge $N$-body models by Li \& Shen (2012). A first spectroscopic study of stars located in the two RCs was performed by de Propris et al. (2011). These authors could not discern any difference in kinematics and metal abundance between the two RCs, which is in agreement with the interpretation that the two RCs represent the same parent population at different distances from the sun. However, these studies were based on optical spectra with relatively low resolution and signal-to-noise ratio $(\mathrm{S} / \mathrm{N})$, from which only an abundance index could be measured. An investigation of the double RC at higher spectral resolution and $\mathrm{S} / \mathrm{N}$ is desirable to foster these conclusions.

In this Paper we present medium-resolution optical spectra of $\sim 400$ bulge stars towards a field at $(l, b)=\left(0^{\circ},-10^{\circ}\right)$, i.e. $\sim 1.4 \mathrm{kpc}$ south of the Galactic plane (assuming a bulge distance of $8.0 \mathrm{kpc}$ ) in the outer part of the bulge. The spectra were obtained with the FLAMES spectrograph at the VLT in GIRAFFE multi-object mode. Our sample includes stars from the tip of the RGB to stars less luminous than the expected bump in the red giant branch (RGB) luminosity function (RGB bump), hence it also includes the two RCs. This is the first study of the two RCs with medium-resolution, high-S/N spectra. We measure from these spectra radial velocities, general metallicities, and abundances of iron and the $\alpha$-elements, and combine this information to obtain a clearer picture of the structure of the outer bulge.

Furthermore, we extensively compare our results with predictions by models of the Galaxy and use these models to infer selection biases and the contamination by non-bulge stars. Recently, Robin et al. (2012) presented a model where two populations co-exist in the bulge region: a bar or pseudo-bulge of high metallicity and small scale height, and a "thick bulge" or classical bulge with a higher scale height, lower metallicity, and higher velocity dispersion. This "Besançon model of the Galaxy" (BGM) explains well the apparent gradient in metallicity that is observed along the minor axis, by a variable proportion of the two populations of different scale height. Here, we use our observed sample as a test case for the new version of the BGM.

The paper is structured in the following way: the sample selection and the observations are presented in Sect. 2; the analysis of the data with the help of COMARCS atmospheric models and spectral synthesis techniques, as well as with the Besançon and TRILEGAL models of the Galaxy, is introduced in Sect. 3; Sect. 4 presents and discusses our results on the radial velocities, metallicities, and $\alpha$-element abundances; in Sect. 5, a comparison of the Galaxy models with 2MASS photometry is done; finally, conclusions are drawn in Sect. 6. 


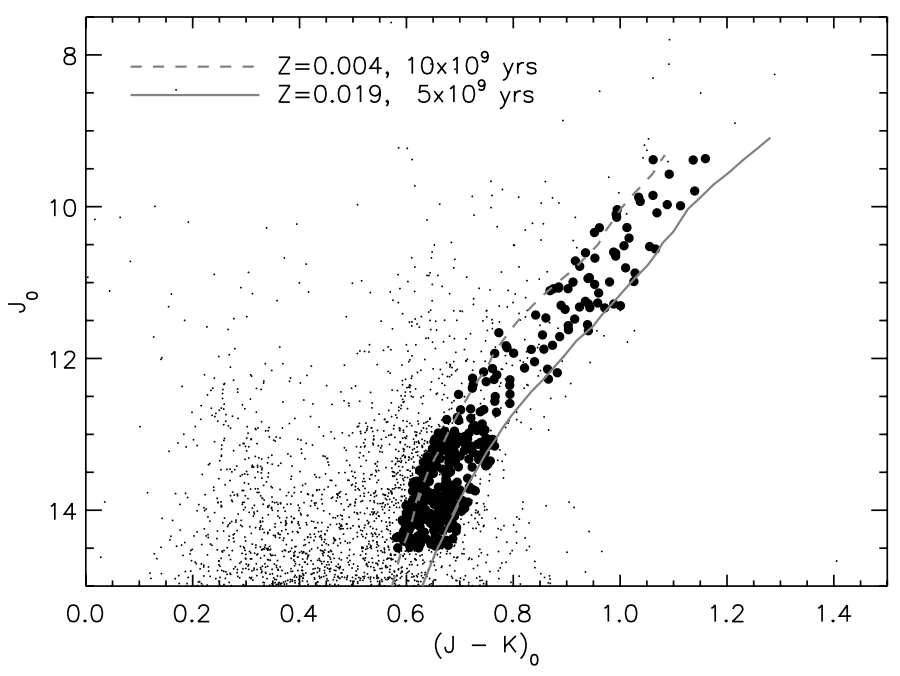

Fig. 1. De-reddened 2MASS colour-magnitude diagram of the $25^{\prime}$ diameter field around $(l, b)=\left(0^{\circ},-10^{\circ}\right)$. Targets observed in the present programme are plotted as black circles, those that have not been observed as black dots. The two RGB isochrones from Girardi et al. (2000) used for the target selection, with ages and metallicities as indicated in the legend, are shown as dashed and solid lines. The isochrones are truncated at the tip of the RGB. The two RCs are the over-densities at $J_{0} \sim 13^{\mathrm{m}} \cdot 2$ and $J_{0} \sim 13^{\mathrm{m}} \cdot 9$.

\section{Sample and observations}

\subsection{Target selection, observations, and data reduction}

Initially, the sample of stars was selected for the study of the evolution of lithium along the bulge RGB (Lebzelter et al. 2012). For a detailed description of the sample selection and the observations we refer to that paper, here we reproduce only the most important points.

The selection of targets was based on data from the 2MASS catalogue (Skrutskie et al. 2006) in a $25^{\prime}$ diameter circle towards the direction $(l, b)=\left(0^{\circ},-10^{\circ}\right)$, which is the centre of the Palomar-Groningen field \#3 (PG3). The sample selection is illustrated in Fig. 1, which shows a colour-magnitude diagram (CMD) of that circular field. The observed targets (black circles) were chosen to fall close to two isochrones from Girardi et al. (2000): $Z=0.004$ and age $10 \times 10^{9}$ years, and $Z=0.019$ (which is $Z_{\odot}$ on the scale used by Girardi et al. 2000) and age $5 \times 10^{9}$ years. These isochrones were used because they cover well the RGB of the bulge. They are not chosen as to reproduce any of the GB's properties, in particular not its age spread. A distance modulus of 14.5 to the GB was adopted. The chosen targets were allowed to have a $\left(J-K_{\mathrm{S}}\right)_{0}$ colour either bracketed by the isochrones, or 0.02 redder or bluer than them. Dereddening of the photometry was done as described in Lebzelter et al. (2012). We find a mean reddening of 0.129 in the $J$-band and $0^{\mathrm{m}} 049$ in the $K_{\mathrm{S}}$-band. Besides the colour criterion, only stars were included that are fainter than $J_{0}=9$. 0 to exclude AGB stars above the tip of the RGB, and stars brighter than $J_{0}=14.5$ to include the RGB bump, which is expected from isochrones at $13.8 \leqslant J_{0} \leqslant 14$. 1 . This region in the CMD will henceforth be referred to as "selection region". Furthermore, all targets were excluded that had fewer than two quality flags "A" in the 2MASS $J H K_{\mathrm{S}}$ photometry, and targets that had another source within $3^{\prime \prime}$ that was not fainter by at least $2 \mathrm{~m} 0$ in the $J$-band than the target itself. Applying all those criteria yielded 514 targets for the observations.

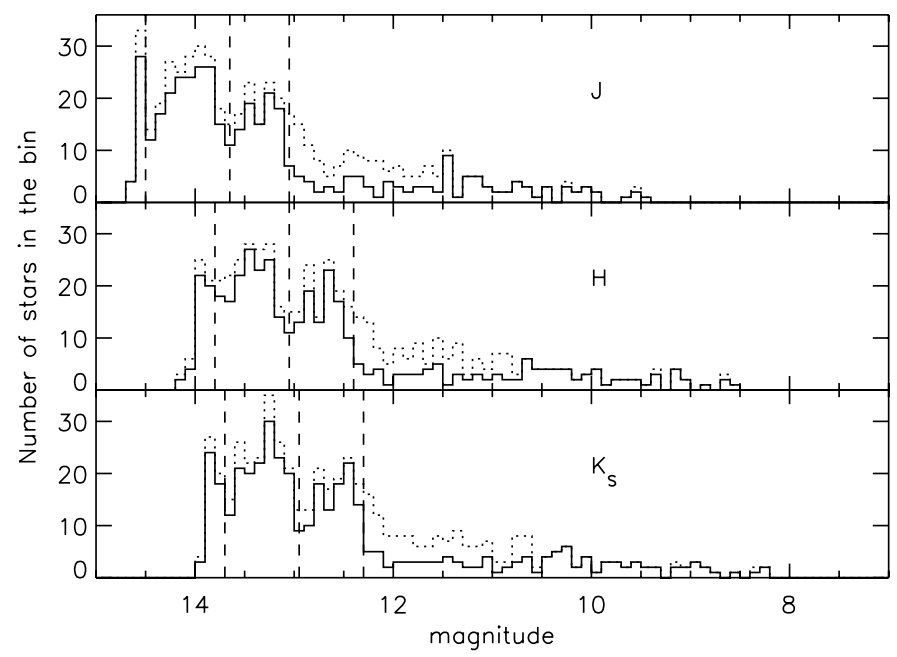

Fig. 2. Histograms of the observed (not de-reddened) 2MASS magnitudes of our sample stars. The dotted line shows the histograms of all stars in the selection region (527 stars, including those that did not fulfil the quality flag criteria to be selected as target), the solid line shows the histogram of the 401 stars for which the radial velocity could be determined. The vertical dashed lines indicate the magnitude limits that were adopted to define the memberships in the two RCs (Sect. 2.2). The double RC is clearly visible in all three bands.

The targets were observed with the FLAMES spectrograph, mounted to the VLT UT2 at Paranal observatory, Chile, in the GIRAFFE configuration. The grating HR15 centred at $665 \mathrm{~nm}$ was used, which gave a spectral resolution of 17000 for a wavelength coverage from 644 to $682 \mathrm{~nm}$. The observations were obtained in service mode in June 2009. Of the initially proposed sample, 401 spectra had sufficiently high quality to measure their radial velocity ( $R V$, see below).

\subsection{Magnitude histograms and the two RCs}

Figure 2 shows histograms of the 2MASS magnitudes of all stars in the selection region (527 stars, dotted line), and of the stars for which the RV could be determined (401 stars, solid line). At intermediate magnitudes $\left(13 \mathrm{~m}^{\mathrm{m}} 0 \leq J_{0} \leq 11 \mathrm{~m} .5\right)$, spectra of only about $40 \%$ of the selected targets are available because one fibre configuration for the stars in this brightness range was not observed. The reduced fraction of actually observed stars will be taken into account in the analysis (Sect. 4.1.1).

Also discernible in Figs. 1 and 2 are the two red clumps (RCs) recently identified by Nataf et al. (2010) and McWilliam \& Zoccali (2010). These structures were not known to us in the design phase of the programme. Initially, the fainter RC at $J_{0} \sim 13.9$ was interpreted as to be the RGB bump, and only the brighter one at $J_{0} \sim 13 \mathrm{~m} 2$ as the red clump. Inspecting Fig. 2, we defined membership of stars in the two RCs as follows. Bright RC: $13.65>J>13.05,13.05>H>12$. 40 , and $12.95>K>12$. 30 ; faint RC: $14.50>J>13.65$, 13 m $80>H>13$ m 05 , and $13.70>K>12^{\mathrm{m}}$. 95 . We found 96 and 150 stars, respectively, which fulfill these criteria. Because it is impossible to ascribe with certainty a given star to either the front or the back arm of the X-structure, the uncertainty of a bulge star's absolute magnitude is about $0 . \mathrm{m} 35$. 


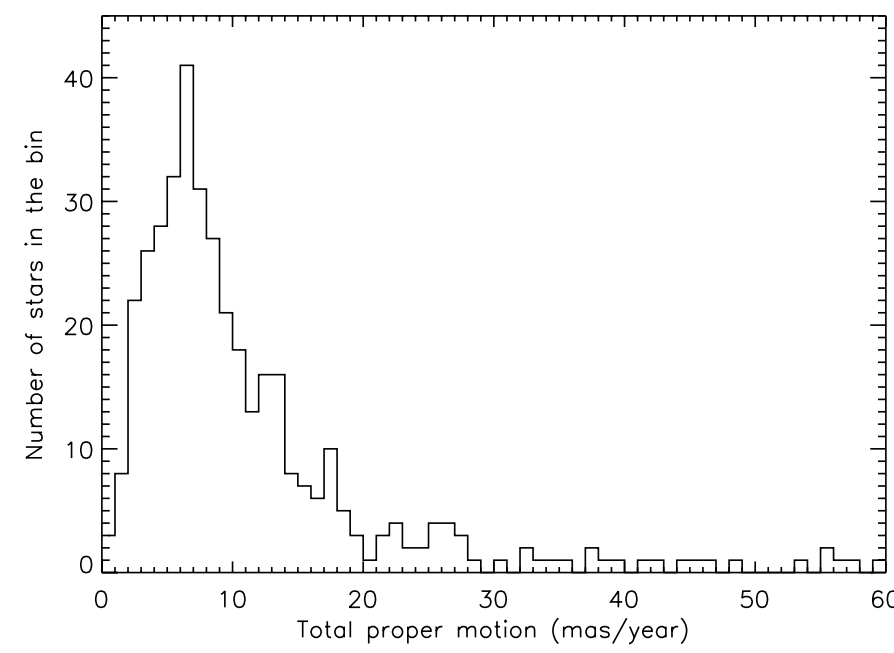

Fig. 3. Histogram of the total proper motion of our sample stars. Stars with a total proper motion larger than 20 mas/year are defined as foreground candidates. Note that four sample stars with a total proper motion larger than 60 mas/year fall outside the plot range.

\subsection{Foreground contamination from proper motions}

An important issue for this kind of study is the foreground contamination of the sample. The proper motion can be used to identify possible foreground stars. We therefore searched the recently published Southern Proper Motion Program catalogue version 4 (SPM4; Girard et al. 2011) for sample stars with large proper motion. All but ten of our sample stars were found in the SPM4 catalogue. In principle, foreground stars could be identified as those stars whose total space velocity clearly exceeds the maximum measured RV, assuming that they are placed at the distance of the GB $(\sim 8 \mathrm{kpc})$. The maximum RV found in our sample is about $300 \mathrm{~km} \mathrm{~s}^{-1}$, which corresponds to a (tangential) proper motion of 7.91 milliarcsec (mas) per year at a distance of $8 \mathrm{kpc}$. Unfortunately, the mean combined error on the proper motion quoted in SPM4 (6.3 mas/yr) is only barely smaller than this value, so with this catalogue it is impossible to probe in detail the proper motions out to the GB. Applying the criterion described above necessarily would return a mixture of bulge and disc foreground stars. Figure 3 shows the distribution of the total (combined) proper motions of our sample. The distribution essentially consists of a broad peak centred on $\sim 7 \mathrm{mas} / \mathrm{yr}$ and a long tail of higher proper motion stars. The tail seems to start at $\sim 20 \mathrm{mas} / \mathrm{yr}$, which is why we decided to assign those stars to the foreground whose combined proper motion is larger than this value. This is also approximately the number that one gets when two times the mean combined error $(2 \sigma)$ is added to the most common total proper motion. Applying this criterion, we found 50 (12.5\%) foreground star candidates, which roughly agrees with the fraction of non-bulge stars estimated from the Galaxy models (see Sect. 3.2.3). The foreground star candidates are marked with an asterisk in Table 3. One star in our sample (\#300) has a particularly large proper motion of more than 200 mas/yr, hence it is probably a nearby K dwarf. In addition, \#143 is probably a foreground $\mathrm{M}$ dwarf.

Unfortunately, there is reason to believe that there are large (systematic) errors in the SPM4 catalogue because the double RC as well as the two peaks in the metallicity distribution (Sect. 4.1.2), two features which are believed to be related to the GB rather than to the disc, are also found among these 50 foreground candidates. Probably there are still many genuine bulge
Table 1. Parameters of the COMARCS model grid.

\begin{tabular}{lcc}
\hline \hline $\log g$ & $J_{0}$ range & $T_{\text {eff }}(\mathrm{K})$ \\
\hline 0.6 & $9^{\mathrm{m}} \cdot 0-10^{\mathrm{m}} \cdot 5$ & $3460,3625,3695,3935$ \\
1.1 & $10^{\mathrm{m}} \cdot 5-11^{\mathrm{m}} 5$ & $3685,3830,4000,4170$ \\
1.6 & $11^{\mathrm{m}} 5-12^{\mathrm{m}} 5$ & $3950,4140,4330,4520$ \\
2.1 & $12^{\mathrm{m}} 5-13^{\mathrm{m}} 5$ & $4275,4450,4500,4525$, \\
& & 4725 \\
2.5 & $13^{\mathrm{m}} 5-14^{\mathrm{m}} 5$ & $4425,4430,4550,4645$, \\
& & $4675,4700,4750,4860$ \\
\hline
\end{tabular}

stars among these foreground candidates, which is why we refrain from excluding these stars from the analysis.

\section{Analysis}

The spectra were analysed with the help of model atmospheres and synthetic spectra, while the results are interpreted with models of the Milky Way galaxy. Both these types of models and the associated analysis are described in the following.

\subsection{COMARCS atmosphere models and COMA synthetic spectra}

For the analysis of the spectra we used model atmospheres calculated with the COMARCS code (Aringer et al. 2009), and spectra based on these models synthesised with COMA (Copenhagen Opacities for Model Atmospheres; Aringer et al. 2009).

A determination of the stellar temperature and surface gravity with a classical equivalent width analysis using only the FLAMES spectra was not possible due to the relatively small spectral range and the dominance of $\mathrm{TiO}$ lines in the cooler targets. Instead, the temperatures of the stars were determined from their 2MASS $\left(J-K_{\mathrm{S}}\right)_{0}$ colour and the effective temperature calibration based on COMARCS models presented in Lebzelter et al. (2012). Hence, the calculations to derive temperatures are consistent with the spectral synthesis applied in this work to obtain abundances in the stars. The surface gravities $(g)$ of the stars were obtained from the $J_{0}$ magnitudes of the stars and the isochrones in Fig. 1, assuming that the stars belong to the bulge RGB. The uncertainty in the distance modulus of \pm 0.35 (Sect. 2.2) introduces an uncertainty in $\log g$ of $\pm 0.16 \mathrm{dex}$, which however has only a minor impact on the uncertainty in the abundances. Furthermore, a generic micro-turbulence of $2.5 \mathrm{~km} \mathrm{~s}^{-1}$, typical of red giant stars, a $\mathrm{C} / \mathrm{O}$ ratio of 0.3 , and a generic overabundance of the $\alpha$-elements $(\mathrm{O}, \mathrm{Ne}, \mathrm{Mg}, \mathrm{Si}, \mathrm{S}, \mathrm{Ar}, \mathrm{Ca}$, and Ti) of +0.2 dex were adopted for the model calculations. This overabundance was adopted because previous spectral abundance studies of bulge stars showed a general enhancement of the $\alpha$-elements of this order of magnitude (e.g. Alves-Brito et al. 2010).

With the temperature, $\log g$, and micro-turbulence fixed in this way, a grid of model atmospheres and associated synthetic spectra was calculated. To keep the grid within reasonable size limits, the RGB was sampled by five different values of $\log g$. For each of these bins, models at various temperatures, in a range applicable for this $\log g$ bin, were calculated. For each combination of $\log g$ and $T_{\text {eff }}$, model atmospheres at five different metallicities $[\mathrm{M} / \mathrm{H}]=1.5,-1.0,-0.5,0.0$, and +0.5 were calculated. The parameters of the model grid are summarised in Table 1.

In the next step, spectra were synthesised for all the models in the grid and convolved with a Gaussian to the resolving power of the observed spectra $(R=\lambda / \Delta \lambda=17000)$. The most 
important line lists used are the one for TiO (Schwenke 1998), CN (Jørgensen 1997), and the atomic lines (VALD; Kupka et al. 1999). This last list was checked and improved by comparing a model spectrum of Arcturus (adopting the stellar parameters and abundances found by Ryde et al. 2010) with the observed high-resolution Arcturus spectrum (Hinkle et al. 2000). The solar abundances listed in Caffau et al. (2008) were adopted as reference scale. The solar metallicity on that scale is $Z_{\odot}=0.0156$.

We emphasise that a particular strength of our method to measure abundances is the consistency of the temperature determination as well as model atmosphere and synthetic spectra calculation, for which the same opacity tables and radiative transfer routines were applied.

\subsubsection{Radial velocity measurement}

The first quantity that was measured from the spectra was the heliocentric radial velocity. This was done with the help of a cross-correlation technique over the whole available wavelength range, using a synthetic spectrum as template. At this step, a model spectrum with similar temperature as the star under investigation was used for the cross-correlation. The abundance of the model spectrum was not adjusted for each star, we simply adopted solar abundances. The RV was successfully determined for 401 sample stars. We estimated a typical error of $\sim 0.5 \mathrm{~km} \mathrm{~s}^{-1}$ on the measured RV from a Gaussian fit to the peak in the crosscorrelation function.

\subsubsection{Determination of the metal abundance}

To determine the general metal abundance from the spectra, we employed a simple interpolation and fitting procedure. First, the value for $\log g$ was chosen according to the $J_{0}$ magnitude of a given star (see Table 1). Then, all model spectra with that $\log g$ and a given metallicity were interpolated to the temperature of the star using spline polynomials. This interpolation was done for every point of the wavelength vector. For the subsequent fitting procedure, the observed spectrum had to be normalised. For the hotter stars $\left(T_{\text {eff }}>3980 \mathrm{~K}\right.$, corresponding to $\left.\left(J-K_{\mathrm{S}}\right)_{0}<0.93\right)$ this was done by adjusting the observed flux to the model spectrum flux around a few continuum points. In the spectra of the cooler stars, lines of TiO are appearing or even dominating the spectrum, which prohibit a safe definition of the continuum; therefore, for these stars the observed spectrum was normalised as to have the same median flux as the model spectrum over the used wavelength range. The wavelength range was limited to 649-680 nm (vacuum wavelength). Finally, the metal abundance was determined by a $\chi^{2}$ minimisation method. The figure of merit to minimise was chosen to be

$\chi([\mathrm{M} / \mathrm{H}])=\frac{1}{N} \sum_{i=1}^{N} \frac{\sqrt{\left(f_{\mathrm{obs}, i}-f_{\text {model }, i}([\mathrm{M} / \mathrm{H}])\right)^{2}}}{f_{\mathrm{obs}, i}}$,

where $N$ is the number of wavelength points, and $f_{\text {obs, } i}$ and $f_{\text {model }, i}$ are the observed and synthetic model flux at wavelength point $i$. Hence, $\chi$ is the mean difference between observed and synthetic model flux, in units of the observed flux. The IDL routine amoeba.pro was used to find the metal abundance $[\mathrm{M} / \mathrm{H}]$ where $\chi$ reaches a minimum. The general metal abundance was successfully determined for 383 sample stars.

A few examples of the typically achieved fit qualities are displayed in Fig. 4. This figure shows a zoom-in on the group of metal lines located between 649 and $651 \mathrm{~nm}$. An advantage

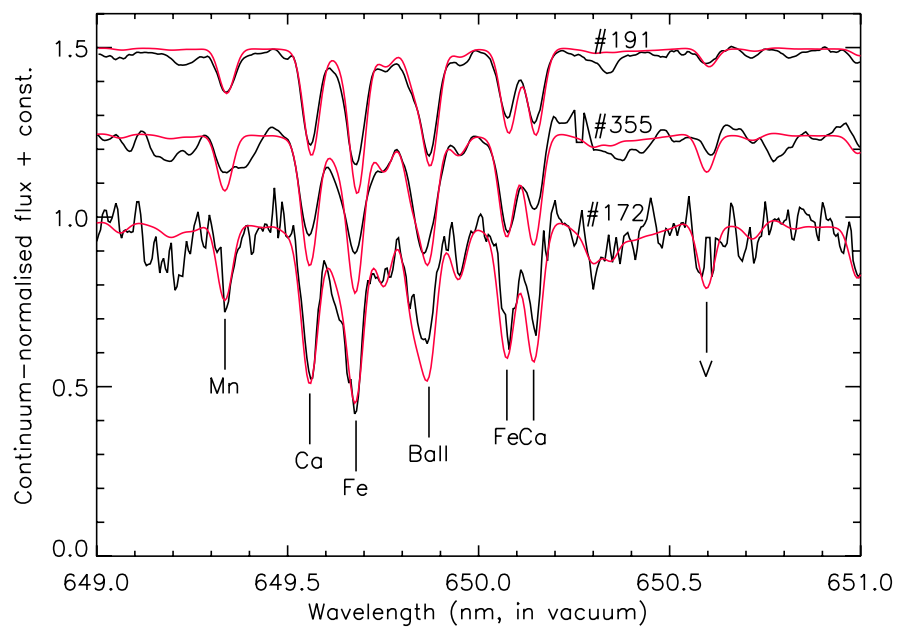

Fig. 4. Typical examples of the fit qualities achieved by our grid interpolation. Observed spectra are plotted as solid black lines, best-fit interpolated model spectra as red lines. From bottom to top: star \#172 $\left(T_{\text {eff }}=4444 \mathrm{~K}, \log g=2.1,[\mathrm{M} / \mathrm{H}]=+0.06, \chi_{\min }=0.0446\right)$, star \#355 $\left(T_{\text {eff }}=4547 \mathrm{~K}, \log g=2.5,[\mathrm{M} / \mathrm{H}]=-0.44, \chi_{\min }=0.0303\right)$, and star $\# 191\left(T_{\text {eff }}=4569 \mathrm{~K}, \log g=2.1,[\mathrm{M} / \mathrm{H}]=-1.05, \chi_{\min }=0.0166\right)$. The spectra of \#355 and \#191 are shifted vertically by 0.25 and 0.5 for clarity. A few prominent metal lines are identified.

of our fitting method is that it also works well at relatively low $\mathrm{S} / \mathrm{N}$ because all the information in the whole wavelength range is taken into account. A good example for this is star \#172 in Fig. 4.

An illustrative check of our metallicity determination is displayed in Fig. 5, which shows a CMD of our sample stars where the colour of the plotting symbol codes the metallicity of each star. Metal-rich stars tend to be more common at the red edge of the selection region, whereas the metal-poor stars tend to be more abundant on the blue edge. This is what can be expected from the evolution along the RGB: at a given brightness, a metalrich star will be cooler, hence redder in $\left(J-K_{\mathrm{S}}\right)_{0}$, than a metalpoor star. This suggests that our methods for temperature and metallicity determination work well. Additional sub-structure around the double $\mathrm{RC}$ is hidden by the high density of stars in that figure; it will be discussed in more detail in Sect. 4.3.

There are a few sample stars for which the fitting routine extrapolated beyond the abundance range covered by the COMARCS model grid. The ten metal-poor outliers clearly have very weak metal lines, indicating a very low metallicity $([\mathrm{M} / \mathrm{H}] \lesssim-1.5)$. With such a low metallicity, these stars possibly belong to the halo population. The two metal-rich outliers are found at the bright and cool end of the sample.

\subsubsection{Determination of the Fe and $\alpha$-abundance}

An estimate of the abundances of iron and the $\alpha$-elements can be achieved with a procedure very similar to the one above. The only difference here is the non-uniform weighting in wavelength. Weight is put only to those wavelength points that are influenced by line absorption by an $\alpha$-element or Fe, respectively. Hence, the $\chi$ value to minimise was altered to

$\chi([\alpha, \mathrm{Fe} / \mathrm{H}])=\frac{1}{N} \sum_{i=1}^{N} W_{i} \frac{\sqrt{\left(f_{\mathrm{obs}, i}-f_{\text {mode }, i}([\alpha, \mathrm{Fe} / \mathrm{H}])\right)^{2}}}{f_{\mathrm{obs}, i}}$,

where $W_{i}$ is the weight function. It is a binary function with value 1 for wavelength points with absorption by $\mathrm{Fe}$ or an 


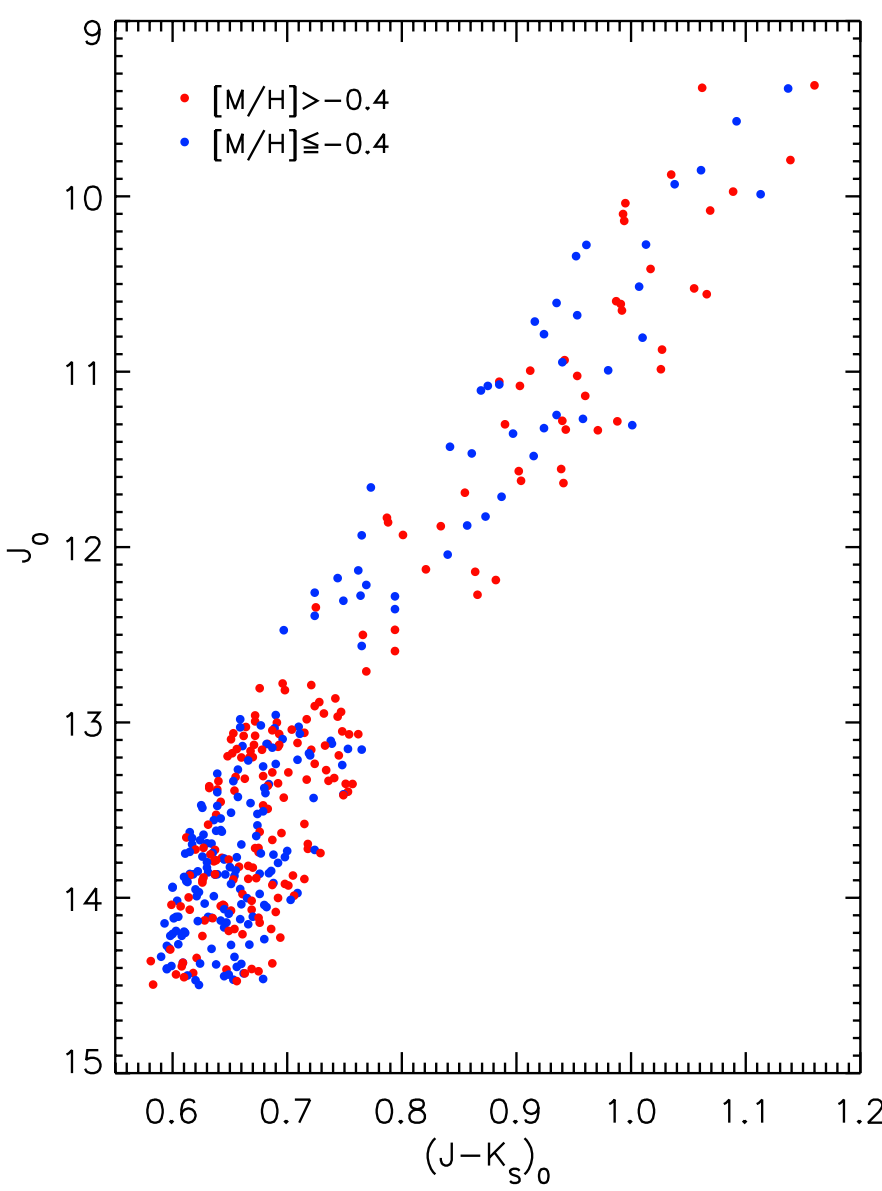

Fig. 5. Colour-magnitude diagram of our sample stars, with the metallicity colour-coded as indicated in the legend. The sample was divided at $[\mathrm{M} / \mathrm{H}]=-0.4$ into two approximately equally large parts. In particular on the upper RGB, metal-rich stars are preferentially located at the red edge of the selection region, whereas metal-poor stars are preferentially at the blue edge.

$\alpha$-element of more than $2 \%$ of the continuum flux, and 0 otherwise. The weight function was calculated from a synthetic spectrum based on the model atmosphere with $T_{\text {eff }}=$ $4450 \mathrm{~K}, \log g=2.1$, and $[\mathrm{M} / \mathrm{H}]=-0.50$, taking into account only the $\alpha$-elements $(\mathrm{O}, \mathrm{Ne}, \mathrm{Mg}, \mathrm{Si}, \mathrm{S}, \mathrm{Ar}, \mathrm{Ca}$, and $\mathrm{Ti})$ or Fe, respectively. The different $\alpha$-elements will not contribute equally to this mean abundance. In particular, $\mathrm{O}, \mathrm{Ne}$, and $\mathrm{Ar}$ have no noteworthy lines in our wavelength range. For the other elements, the relative weight on the average $\alpha$ abundance is: Ca:Ti:Si:Mg:S = 1.000:0.751:0.400:0.027:0.009. Hence, the $\alpha$-abundance is dominated by the individual abundances of $\mathrm{Ca}, \mathrm{Ti}$, and $\mathrm{Si}$. The interpolation was done between the grid points at $[\alpha / \mathrm{H}]=(-1.3,-0.8,-0.3,+0.2,+0.7)$ and $[\mathrm{Fe} / \mathrm{H}]=(-1.5,-1.0,-0.5,0.0,+0.5)$, respectively. The fitting routine did not converge for all stars. Eventually, $\alpha$-abundances were determined for 368 stars, and Fe abundances for 363 stars. The determination of the iron and $\alpha$-abundance failed in particular for many of the cool, TiO-dominated stars. The Fe abundance is a very good tracer of the general metal abundance: the mean of $[\mathrm{M} / \mathrm{H}]-[\mathrm{Fe} / \mathrm{H}]$ is $-0.13 \mathrm{dex}$, with a standard deviation of 0.12 dex; the standard deviation decreases to only $0.07 \mathrm{dex}$ among the sample stars fainter than $J_{0}=12$. 7 .

All measured abundances and radial velocities can be found in Table 3.

\subsubsection{Systematic errors}

A check of our relatively simple methods for the metallicity and abundance determination was done by applying it to the spectrum of Arcturus. For this star, our method yields a metallicity of $[\mathrm{M} / \mathrm{H}]=-0.72$, and $[\mathrm{Fe} / \mathrm{H}]=-0.92$. If dedicated model atmospheres with the parameters from Ryde et al. (2010) are used $\left(T_{\text {eff }}=4280 \mathrm{~K}, \log g=1.7\right)$, i.e. if the grid interpolation in temperature is avoided, $[\mathrm{M} / \mathrm{H}]$ increases to -0.69 . Models without an $\alpha$-enhancement (i.e. scaled solar abundances) yield $[\mathrm{M} / \mathrm{H}]=-0.64$. A search on the Simbad database ${ }^{1}$ shows that most studies find $[\mathrm{Fe} / \mathrm{H} \sim-0.50$ for Arcturus.

To back-up the existence of a systematic abundance offset, we made a blind test with four stars from the sample of Gonzalez et al. (2011a), which cover a range of almost $1 \mathrm{dex}$ in $[\mathrm{Fe} / \mathrm{H}]$. The spectra of the stars in the FLAMES HR15 setting were kindly provided by Gonzalez (priv. comm.) and their stellar parameters as determined in Zoccali et al. (2008) were communicated, but not their individual abundances. The same fitting routine as for our sample stars were applied to the spectra. The results were then compared to those of Gonzalez et al. (2011a). The Fe abundances derived with our method were systematically lower by 0.19 to 0.29 dex than those of Gonzalez et al. (2011a).

Systematic abundance offsets of 0.2 to 0.3 dex between equivalent width measurements and $\chi^{2}$ minimisation techniques (in the sense that the $\chi^{2}$ minimisation gives lower abundances) have also been reported by the RAVE survey team (Zwitter et al. 2008; Boeche et al. 2011), in particular for giant stars. No explanation as for the possible origin of this offset is given in the literature, neither did we attempt to track down its cause. One explanation could be that the classical equivalent width measurement uses only relatively few lines, selected to be unblended, whereas the $\chi^{2}$ minimisation uses many more lines, even blended and partially saturated ones. While the reasons are only speculative at this point, we decided to add 0.2 dex to all abundances determined here. This does not only yield better agreement with the comparison stars of Gonzalez et al. (2011a) and Arcturus, but, as we will see below, gives very satisfactory agreement with other studies of abundances in GB stars. The thereby corrected abundances will be used henceforth in the analysis of our data set and are also reported in Table 3. Despite the systematics in the abundance scale, we are nevertheless confident that our abundance determination is reliable in the relative sense within the sample. Furthermore, we decided to base our analysis mainly on the $[\mathrm{M} / \mathrm{H}]$ measurement because it is available for a few more stars, in particular more of the bright stars. The results would remain qualitatively unchanged if $[\mathrm{Fe} / \mathrm{H}]$ was used instead.

\subsubsection{Uncertainty estimate}

The uncertainty in the determination of the metallicity was estimated for six representative sample stars covering a range in temperature, metallicity, brightness, and $\mathrm{S} / \mathrm{N}$. This was done mainly by varying the free parameters within their uncertainties, one at a time while keeping the others constant, and then repeating the fitting process. Uncertainties arising from the determination of temperature and $\log g$, as well as in the continuum placement, were taken into account.

The uncertainty in the effective temperature was determined from the uncertainty on $\left(J-K_{\mathrm{S}}\right)_{0}$ by adopting the uncertainties in the $J$ - and $K_{\mathrm{S}}$-band magnitudes given in the 2MASS catalogue. For the bright (cool) stars this uncertainty is between 60

1 http://simbad.u-strasbg.fr 
and $100 \mathrm{~K}$, while for the faint (hot) ones it is between 130 and $200 \mathrm{~K}$. However, this does not mean that the temperature has a lower impact on the $[\mathrm{M} / \mathrm{H}]$ determination for the bright and cool stars, just on the contrary: because their spectra are dominated by the very temperature-sensitive $\mathrm{TiO}$ bands, a change in temperature has the largest impact on their metallicity determination. Uncertainties up to $0.27 \mathrm{dex}$ from the temperature uncertainty alone were found, but for most stars it is in the range 0.09 to 0.18 dex.

The uncertainty coming from the $\log g$ estimate was determined by doing the interpolation and fitting with a group of model spectra adjacent in $\log g$ to the one that would be attributed to a given star based on its $J_{0}$ magnitude. We found that the $\log g$ estimate contributes $0.06-0.10$ dex of uncertainty in the $[\mathrm{M} / \mathrm{H}]$ determination. This should already include the uncertainty coming from the fact that we interpolate model spectra to the temperature of the stars, rather than using model spectra calculated for the exact temperature of every star.

Finally, the uncertainty introduced by the continuum placement was estimated by varying the normalisation factor within its uncertainty range, derived from the scatter of the pixels in the continuum points. This uncertainty mainly depends on the $\mathrm{S} / \mathrm{N}$ of the spectrum, and contributes another $0.02-0.04$ dex to the uncertainty in $[\mathrm{M} / \mathrm{H}]$. Thus, for most stars the combined uncertainty in $[\mathrm{M} / \mathrm{H}]$ is in the range $0.11-0.21 \mathrm{dex}$, but it can be as high as 0.30 dex for the cool stars dominated by TiO absorption. The uncertainties in the iron and $\alpha$-element abundances are very similar.

\subsection{The Besançon and TRILEGAL models of the Galaxy}

\subsubsection{The Besançon model}

The Besançon Galaxy Model (BGM) is based on assumptions on the scenario of formation and evolution of four main stellar populations of the Milky Way (Robin et al. 2003). It allows to simulate the stellar content in any given line of sight, and for each simulated star the photometry, kinematics, and metallicity are computed. For each population, the main assumtions are: a star formation-rate history, age, and initial mass function, which allow to generate a distribution function in absolute magnitude, effective temperature, and age of the stars. These assumptions are confined by observations, such as the stellar content in the solar neighbourhood, remote star counts, and photometry in the visible and near-infrared. Density laws are assumed and controlled by dynamical principles (Bienaymé et al. 1987) for each population and are tested by means of photometric star counts. The model has been extensively compared with 2MASS data which allow to constrain the thin disc parameters quite well, such as the scale length, scale height, the inner disc hole, and the warp (Reylé et al. 2009). However, some model parameters are not well constrained yet, such as for the thick disc population. When a parameter is not yet well constrained from available data, such as metallicity gradients for example, a mean value from the literature is assumed, or a value expected from standard scenario of formation. For example, thick disc parameters are still controversial: its density parameters such as the local density and the scale height are degenerated when obtained by model fitting from high galactic latitude fields (Robin et al. 2012). However, in the direction considered here, the thick disc population remains marginal. We conservatively use here the thick disc parameters determined from high latitude counts by Reylé \& Robin (2001).

Once density functions and distribution functions are obtained, the simulated stars are generated in the whole Galaxy divided in volume elements. A number of improvements have been made to the model since the one presented in Robin et al. (2003). The mean metallicity and dispersion about the mean for each age bin in the thin disc are now taken from Haywood (2008), rather than from Twarog (1980). The model also includes a 3D extinction map (Marshall et al. 2006) and photometric errors are added for comparison with observations.

Recently, a new model of the bulge/bar region has been proposed by Robin et al. (2012), which we will use here to interpret and compare to our observed data. The new model includes a bar and a "thick bulge". In the following, this latter population will be referred to as the bulge when discussing the BGM. The bar is the most massive component, which dominates the stellar content at low latitudes. The bulge is longer and thicker and gives a contribution at intermediate latitudes where the bar starts to be less prominent. The main bar population has a mean metallicity close to solar, while the bulge population is found to be more metal-poor, with a metallicity of the order of -0.4 to -0.5 dex. The mean metallicties and dispersions of these two populations have been roughly estimated from the observed MDF by Zoccali et al. (2008). It should be better constrained in the future using larger samples and more lines of sights in the inner and outer bulge.

A large hole in the central region causes the thin disc to reach its maximum density at about $2.5 \mathrm{kpc}$ from the Galactic centre. The kinematics of the thin disc follow empirical estimates from Hipparcos (Gomez et al. 1997), while for the thick disc it is taken from proper motion data from Ojha et al. (1996), and for the halo from Norris et al. (1985), as in Robin et al. (2003). In the version presented here, the bar kinematics are taken from the dynamical model of Fux (1999). The bulge is found to have a larger velocity dispersion, close to the one of the halo (Robin et al., in prep.).

The fact that the bar population has a smaller velocity dispersion, a higher metallicity, and a smaller scale height than the bulge allows to explain well the vertical gradient of metallicity seen in bulge fields along the minor axis (Zoccali et al. 2008). Babusiaux et al. (2010) already suggested that the origin of the metallicity gradient could be a varying mix of these two populations. The bar and bulge populations in the BGM do not have an intrinsic metallicity gradient. This model also explains well the presence of double clumps at medium latitudes, as seen in Nataf et al. (2010), McWilliam \& Zoccali (2010), and Saito et al. (2011). This derives from the fact that the bar flares from inside to outside by about $30 \%$. This flare can be due to resonances in the bar that trap thin disc stars and scatter them to higher scale height.

\subsubsection{The TRILEGAL model}

The TRILEGAL model was developed by Girardi et al. (2005) and is a population synthesis code for simulating the stellar photometry of any field in the Milky Way galaxy. The model has proven to well reproduce number counts, amongst others, in all three pass-bands of the 2MASS catalogue, with errors smaller than $\sim 30$ percent (Girardi et al. 2005). The bulge component was introduced in TRILEGAL only later by Vanhollebeke et al. (2009), who also calibrated its stellar population and metallicity distribution using photometry from the 2MASS and OGLE-II surveys of red clump stars. The input MDF for the bulge component in TRILEGAL was obtained by Vanhollebeke et al. (2009) with the help of a fitting procedure to the RC star distribution, by applying shifts to the photometric MDF of Zoccali et al. (2003). The best fit was obtained with a shift of +0.3 dex. Note that Vanhollebeke et al. (2009) used fields that are considerably 
closer to the Galactic plane than our field, at most $5.91^{\circ}$ south of the plane.

Their best-fit bulge model is provided as the default in the interactive web interface to TRILEGAL ${ }^{2}$, which we also used here to simulate the stellar content of our FLAMES field. Other important ingredients that we adopted included: a binary fraction of 0.3 with mass ratios between 0.7 and 1.0 ; an exponential extinction law with an extinction value at infinity equal to the one used to deredden the observed photometry ( 0.526 in the $V$-band); a thin and a thick disc, both with squared hyperbolic secant density distributions; and a halo with an oblate $r^{1 / 4}$ spheroid density distribution. For all these components, the default parameters offered on the web interface were adopted. The TRILEGAL model does not provide for the kinematics of its simulated stars, hence a comparison with our observations was not possible in this respect.

A circular field of $25^{\prime}$ diameter centred on the point $(l, b)=$ $\left(0^{\circ},-10^{\circ}\right)$, equal to the area from which the observed targets were selected, was simulated with both models. An exception here is that for the BGM we also calculated ten simulations with identical parameters, to decrease the Poisson noise mainly for the comparison with the observed RVs. For all subsequent comparisons between spectroscopic observations and the Galaxy models, the same photometric selection criteria were applied to the models as to the observed sample (Sect. 2.1). In Sects. 4 and 5, we will confront the model predictions with our observations in terms of kinematics, abundances, and photometric properties.

\subsubsection{Inferring metallicity biases, foreground contamination, and mass distribution with the Galaxy models}

The Galaxy models can be very useful to infer a number of important properties of the observed sample, in particular metallicity biases imposed by our photometric selection criteria, (foreground) contamination by non-bulge stars, and the distribution of initial masses.

The location of a bulge star in a CMD, and hence its probability to fall in our selection region, is determined by a complex interplay of metallicity, distance, age, reddening, and photometric errors. The ratio of the input MDF for the bulge (and bar) component to the MDF of the stars selected with our procedure reveals any such metallicity biases. An inspection of this ratio for the BGM simulation shows that there exists a small bias against intermediate metallicities $([\mathrm{M} / \mathrm{H}] \sim-0.4)$. This is probably caused by the different distance distributions of the bulge and the bar component in the BGM: the bulge component, which contributes most stars in this intermediate to low metallicity, is spread out between 2 and $14 \mathrm{kpc}$ distance from the sun, whereas the bar, which is centred around solar metallicity, is much more concentrated around $7 \mathrm{kpc}$ distance. Many of the nearby bulge stars thus do not fall in the selection region, which causes this small bias against intermediate metallicity stars. In fact, an inspection of the distance distribution shows that our selection procedure is relatively efficient in picking up stars at distances between 5 and $11 \mathrm{kpc}$.

The TRILEGAL model includes a single bulge component, with a distance distribution centred on $7 \mathrm{kpc}$. Hence, the metallicity bias inferred from this model somewhat differs from the one found from the Besançon model. The ratio of input MDF to the MDF in the selection region is relatively flat in a broad range from $[\mathrm{M} / \mathrm{H}] \sim-0.4$ to +0.5 , but gradually decreases to

\footnotetext{
${ }^{2}$ http://stev.oapd.inaf.it/trilegal
}

lower metallicities. This is also what one would expect from a bulge population with a relatively small distance spread: because low-metallicity stars evolve along the RGB at relatively blue $J-K_{\mathrm{S}}$ colours, in particular on the upper RGB, they would be missed by our selection procedure (along relatively metalrich isochrones) with a higher probability. Consistent with this expectation, we find in both models that the metal-poor stars in the selection region are preferentially at larger distances than the metal-rich ones because the blue, metal-poor stars are shifted into the selection region when they are at large distances.

Both the Besançon and TRILEGAL model allow to assign a simulated star to one of the stellar components of the Milky Way, by which means we estimated the fraction of genuine bulge stars in our sample. The selection of stars from the Besançon model yielded 688 stars, of which $333(48.4 \%)$ belong to the thick bulge, $216(31.4 \%$ ) to the bar (hence $79.8 \%$ of bulge/bar stars in total), $72(10.5 \%)$ to the thin disc, $64(9.3 \%)$ to the thick disc, and $3(0.4 \%)$ to the halo. On the other hand, in the TRILEGAL model 509 stars fall in the selection region, of which $479(94.1 \%)$ belong to the bulge, $21(4.1 \%)$ to the thin disc, 9 $(1.8 \%)$ to the halo, and none to the thick disc. From this estimate we conclude that a large fraction $(\gtrsim 80 \%)$ of our sample stars are genuine bulge stars. Most of the foreground contamination comes from the thin disc, while the halo contamination should be very small. The fraction of non-bulge stars agrees reasonably well with the fraction of foreground star candidates selected on basis of their proper motion $(12.5 \%)$ in Sect. 2.3. Most notably, applying the same proper motion selection criterion of $>20$ mas/yr to the simulated stars in the Besançon model retrieves 52 foreground star candidates, in excellent agreement with the number we found from the SPM4 catalogue. Of these 52 stars, 51 are thin disc stars and one is a thick disc star.

The distribution of initial masses of the simulated stars turns out to be relatively sharply peaked in both models. In the Besançon model the mean mass is $1.05 M_{\odot}$ with a standard deviation of $0.11 M_{\odot}$, whereas in TRILEGAL the mean is at $1.13 M_{\odot}$ with a standard deviation of $0.14 M_{\odot}$. Genuine bulge stars have an even narrower distribution: $1.07 \pm 0.05 M_{\odot}$ in the Besançon model and $1.15 \pm 0.09 M_{\odot}$ in the TRILEGAL model. The most massive stars selected from the simulated data have 1.39 and $1.59 M_{\odot}$ in the Besançon and TRILEGAL model, respectively. Hence, we are confident that our sample contains mostly lowmass $\left(M \sim 1.1 M_{\odot}\right)$ red giant stars located in the GB.

\section{Results and discussion}

\subsection{The observed metallicity distribution function}

\subsubsection{Whole sample}

The MDF of our sample stars is displayed in Fig. 6. Two versions are plotted: the raw MDF (solid line), and a MDF corrected for the incompleteness in some brightness (and hence colour) ranges in our sample. To correct for this, we applied a bootstrapping method similar to method ii) used by Zoccali et al. (2008). In this method, stars are drawn randomly until a flat ratio of observed to available stars is reached. Zoccali et al. (2008) considered this ratio as a function of colour. Instead, we chose to consider it as a function of brightness because our targets were grouped for observations according to their brightness, where one of the scheduled settings for the intermediate brightness group was not observed. However, since our selection region diagonally crosses the CMD, any under-sampling in colour bins will be effectively corrected for by this method. 


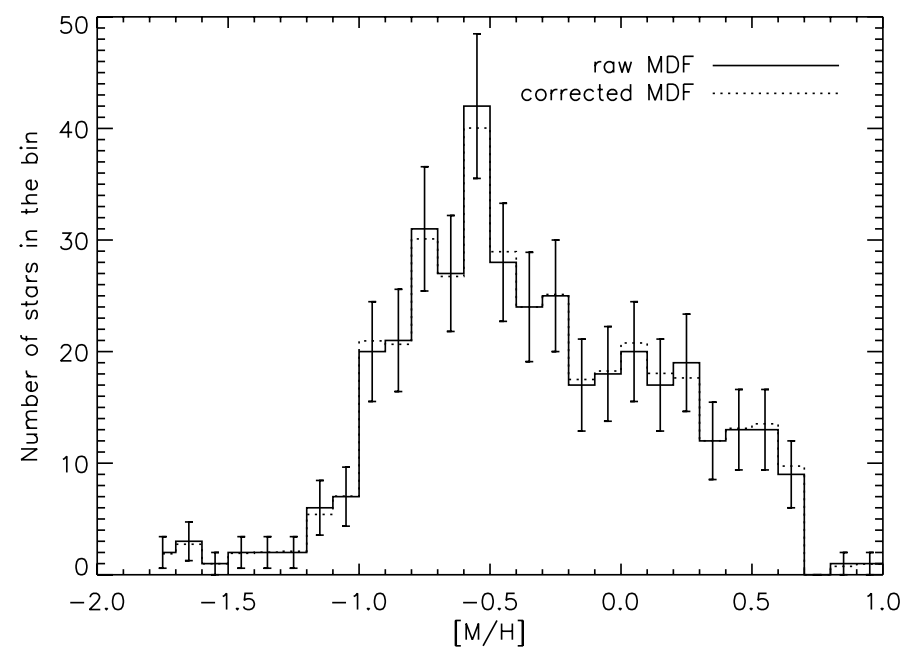

Fig. 6. Metallicity distribution function of all 383 sample stars with determined metallicities. The solid line is the raw MDF, while the dotted line is an MDF corrected for the non-uniform fraction of observed vs. available targets as a function of magnitude, using a bootstrapping method. Poissonian noise error bars are also shown for the raw MDF.

Two-hundred such randomly drawn MDFs were created and averaged, the result of this procedure is plotted as dotted line in Fig. 6. The corrected MDF is only marginally different from the raw MDF, hence we conclude that our sample well represents the metallicities of stars in the whole selection region.

The MDF is broad with a clear peak at $[\mathrm{M} / \mathrm{H}] \sim-0.55$. The median, mean $^{3}$, and standard deviation of the MDF are $-0.41,-0.34$, and 0.51 dex. This mean metallicity agrees well with what Zoccali et al. (2008) found: for fields at $b=-6^{\circ}$ and $b=-12^{\circ}$ they found $\langle[\mathrm{Fe} / \mathrm{H}]\rangle=-0.21$ and -0.29 , respectively. Interpolating between these fields to $b=-10^{\circ}$ yields a mean of -0.26 . Note, however, that Zoccali et al. (2008) estimated a large contamination by thin and thick disc stars in their sample at $b=-12^{\circ}$, which may have a considerable impact on the mean metallicity of this field. Whereas Zoccali et al. (2008) used a box in an $I$ vs. $V-I$ CMD to select their targets, we used a selection region along isochrones, a strategy that is probably much more discriminant against thin and thick disc contaminants. Johnson et al. (2011a) derived spectroscopic iron abundances of RGB and RC stars in two fields at $(l, b)=\left(0^{\circ},-8^{\circ}\right)$ and $(l, b)=\left(-1^{\circ},-8.5^{\circ}\right)$. The mean $[\mathrm{Fe} / \mathrm{H}]$ found for these fields are -0.34 and -0.27 , respectively. These authors also derive photometric metallicities for the latter field and find a median of $[\mathrm{Fe} / \mathrm{H}]=-0.34$. Also Gonzalez et al. (2011b) derived photometric metallicities along the GB minor axis and find $\langle[\mathrm{Fe} / \mathrm{H}]\rangle=-0.36$ in a $30^{\prime} \times 30^{\prime}$ field around $b=-8^{\circ}$. These values all agree nicely with one another, and with our result for the field at $b=-10^{\circ}$.

The MDF in Fig. 6 exhibits a relatively sharp cut-off at $[\mathrm{M} / \mathrm{H}] \sim-1.0$. This could be related to the use of an isochrone with $Z=0.004$ (corresponding to $[\mathrm{M} / \mathrm{H}]=-0.68$ ) for defining the blue edge of the selection region. As suggested by the TRILEGAL model (Sect. 3.2.3), there probably is a bias agains metal-poor stars in the selection, which affects the size and shape of the metal-poor peak. This metallicity selection bias will be smoothed by the distance spread of the bulge stars. However, it is hard to quantify if this sharp cut-off is a real feature or

\footnotetext{
${ }^{3}$ Doing the averaging on a linear scale, which is the mathematically correct way, the mean is $[\mathrm{M} / \mathrm{H}]=-0.05$, converted back to the logarithmic scale.
}

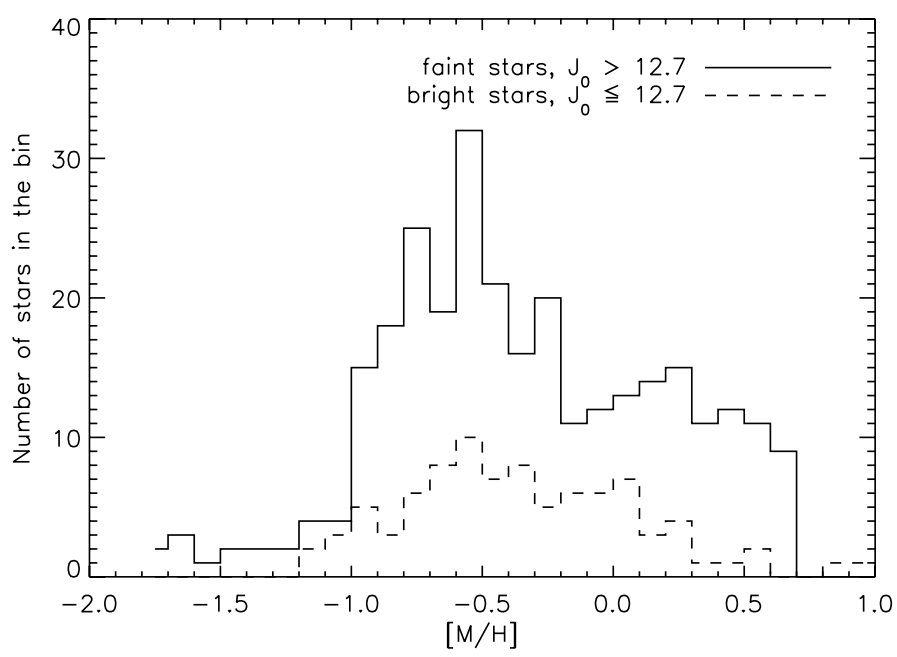

Fig. 7. Metallicity distributions of bright $\left(J_{0} \leq 12^{\mathrm{m}}\right.$.7 dashed line) and faint $\left(J_{0}>12 \mathrm{~m}\right.$. , solid line) sample stars. The metal-poor and metal-rich populations are more clearly separated among the faint sample stars.

an effect of the selection bias because of the lack of stars with $[\mathrm{M} / \mathrm{H}] \lesssim-1.0$ in the input MDF of the models.

\subsubsection{Bright and faint sample stars}

It becomes clear already from Fig. 6 that there are possibly two peaks in the MDF: one at $\sim-0.55$ and one at $\sim 0.2$. This doublepeak structure becomes even more apparent if bright and faint stars are considered separately (Fig. 7). The cut between bright and faint stars was introduced at $J_{0}=12 \mathrm{~m}$. 7 , which should safely exclude RC stars from the bright sub-sample. The motivation to introduce a brightness cut is two-fold: first, the uncertainty in the metallicity is larger $(\sim 0.30$ dex $)$ for the coolest and brightest sample stars. Thus, with a brightness cut we exclude stars with the most uncertain metallicity determination. Second, a brightness cut is equivalent to a cut in evolutionary state, at least when the distance spread within the bulge of $\sim 0.7$ is disregarded. Hence, with our sample we can check whether the MDF varies with evolutionary state or not.

The most striking feature in Fig. 7 is that among the faint sample stars (those with $J_{0}>12.7$ ) the dichotomy in metallicities becomes even clearer than in the complete sample. To make this dichotomy more quantitative we performed a decomposition by Gaussian mixture models. The parameter space of two Gaussian mixture models was explored using a Markov Chain Monte Carlo simulation and a maximum-likelihood approach to define the best-fit parameters (for details on the code see Nataf et al. 2011, for an in-depth explanation of MCMCs see Johnson et al. 2011b). The chosen confidence level is $68 \%$. Stars with $[\mathrm{M} / \mathrm{H}]<-1.4$ were omitted from the fit because we think these stars might be halo contaminants. The results of this Gaussian decomposition are presented in Fig. 8. We find mean metallicities of the populations of $[\mathrm{M} / \mathrm{H}]=-0.57 \pm 0.03$ and $[\mathrm{M} / \mathrm{H}]=+0.30 \pm 0.04$, and dispersions of $0.27 \pm 0.02$ and $0.28 \pm 0.03 \mathrm{dex}$ for the metal-poor and metal-rich population, respectively. The metallicity difference between the two populations, the systematically and statistically more robust figure, is $\Delta[\mathrm{M} / \mathrm{H}]=0.87 \pm 0.03$. The number of stars belonging to those peaks is $198 \pm 12$ and $88 \pm 12$, respectively, yielding a fraction of the metal-rich population of $(30.8 \pm 4.2) \%$. The TRILEGAL model suggests that there could be a selection bias against metalpoor stars (Sect. 3.2.3); if true, then the fraction of metal-poor 


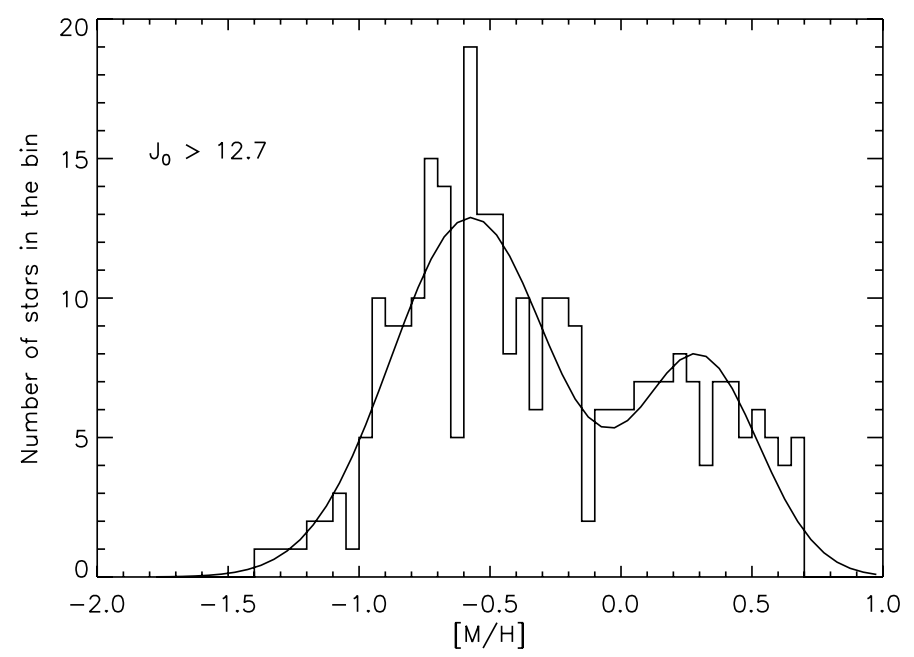

Fig. 8. Metallicity distribution of the faint sample stars $\left(J_{0}>12.7\right)$ together with the two best-fitting Gaussian mixture models. The metallicity distribution in this brightness range is clearly bimodal.

stars at this Galactic latitude has to be regarded as a lower limit, and that of the metal-rich ones as an upper limit.

\subsubsection{Comparison with the literature}

Recently, MDFs of bulge stars were presented by Hill et al. (2011) and Bensby et al. (2011), both of which find a doublepeaked MDF. Hill et al. (2011) presented $[\mathrm{Fe} / \mathrm{H}]$ measurements for 219 bulge red clump stars in Baade's window $\left(b=-4^{\circ}\right)$, derived also from FLAMES/GIRAFFE spectra. Their Gaussian decomposition yields a broader peak at $[\mathrm{Fe} / \mathrm{H}] \approx-0.30$ and a relatively narrow peak at $[\mathrm{Fe} / \mathrm{H}] \approx+0.32$, respectively. On the other hand, Bensby et al. (2011) measured from high-resolution UVES/VLT spectra the metallicities of micro-lensed dwarf and sub-giant stars scattered throughout the Galactic bulge at latitudes between $b=-1^{\circ}$ and $-5^{\circ}$, at various longitudes. The dwarfs show also a clearly bi-modal distribution with peaks at $[\mathrm{Fe} / \mathrm{H}] \approx-0.60$ and $\approx+0.3$. Our result is in excellent agreement with that of Bensby et al. (2011), even though our field is several degrees (corresponding to $\sim 1 \mathrm{kpc}$ ) away from the dwarfs analysed by these authors. Only the metal-poor population identified by Hill et al. (2011) has a higher metallicity by $\sim 0.3$ dex. Unfortunately, the number of analysed bulge dwarfs is still too low to reliably estimate the widths of the two peaks in their MDF. In contrast to the results of Hill et al. (2011), the peaks in the MDF found in our sample have very similar widths. In general, there are interesting analogies between our results and those of Bensby et al. (2011) and Hill et al. (2011), and we conclude that there are two populations present in the GB, separated by $0.6-0.9$ dex in metallicity. The small differences might be a result of the different selection procedures, the difference in evolutionary state of the samples, and the different analysis methods applied in the studies.

\subsection{Evolutionary effects on the MDF}

Another interesting feature of Fig. 7 is the probable lack of metal-rich stars among the bright sample stars $\left(J_{0} \leq 12\right.$. 7$)$. In the metallicity range $+0.1 \leq[\mathrm{M} / \mathrm{H}] \leq+0.7$, eleven stars are bright and 72 stars are faint $(13.3 \pm 4.3 \%$ bright stars, the uncertainty being calculated from Poissonian noise), whereas in the range $-0.5 \leq[\mathrm{M} / \mathrm{H}] \leq+0.1$ the fraction is $29.5 \pm 5.4 \%$

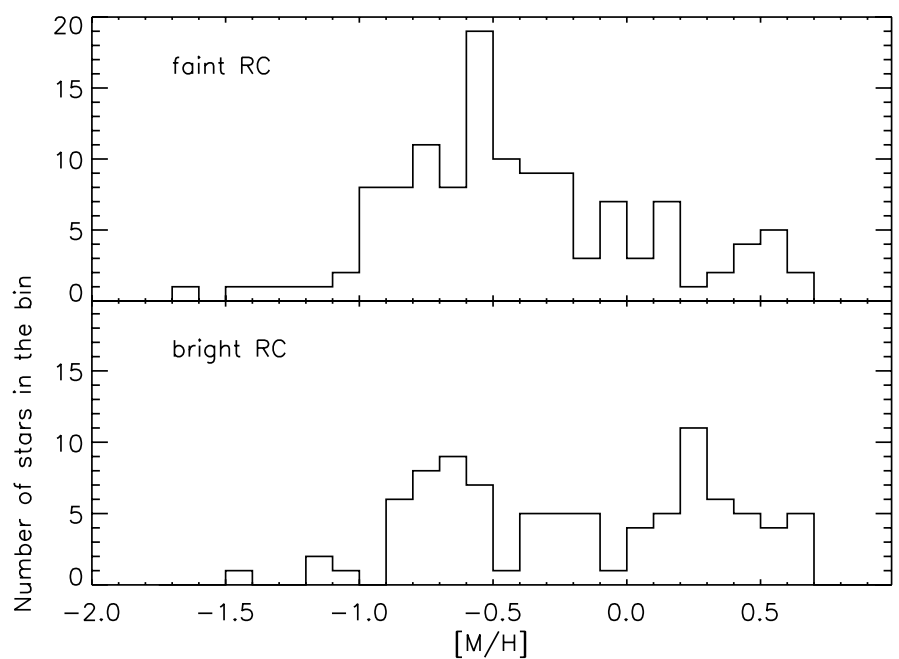

Fig. 9. Metallicity distribution of stars belonging to the faint (upper panel) and bright (lower panel) RCs, respectively.

(39 bright and 93 faint stars). The fraction of bright stars in the whole sample is $22.9 \pm 2.7 \%$ (92 bright and 309 faint stars). This reduced fraction of bright stars among the metal-rich stars could be caused by a selection effect. We therefore inspected the fraction of bright stars in the selection region of the Galaxy models as a function of metallicity to check for differences in the metallicity bias between faint and bright RGB stars (i.e. less and more evolved stars). However, no such trend is found, which suggests that the metallicity biases equally apply to faint and bright stars.

Apparently, the shape of the MDF depends on the evolutionary state of the sample stars one is considering. The simplest explanation for this finding is that the metal-rich stars do not evolve all the way from the main sequence up to the RGB tip or the early AGB, but get "lost" somewhere on the way (e.g. due to enhanced mass loss; Castellani \& Castellani 1993). A similar scenario was put forward by Chiappini et al. (2009) to explain the shift of 0.3 dex to lower values in the planetary nebulae oxygen abundance distribution with respect to the distribution of giant stars. Because of the still relatively small number of stars in the present sample this conclusion has to be taken with some caution. Also, some (presumably metal-rich) stars on the red side of the selection region have not been observed in the present programme (see Fig. 1). However, in Sect. 4.7.3 we will present another piece of evidence from the radial velocity dispersion of evolved stars that strongly supports the scenario of metallicity-dependent mass loss.

\subsection{The metallicity of the red clump stars: is the double $R C$ only connected to the metal-rich population?}

Figure 9 shows the MDF of the bright and faint RC, with the definition of the two RCs as introduced in Sect. 2.2. It is clear from that figure that the faint $\mathrm{RC}$ has a much more prominent low-metallicity peak than the bright $\mathrm{RC}$, which in turn shows both peaks. Their mean metallicities are $[\mathrm{M} / \mathrm{H}]=-0.18$ and -0.41 , respectively. This would suggest that the two RCs have intrinsically different metallicities, in contradiction to findings by de Propris et al. (2011), and would therefore not represent the same underlying population of stars. However, we believe that this seemingly different mean metallicity is actually caused by our selection procedure. Because of the way how we selected the stars along two isochrones, which become redder 
S. Uttenthaler et al.: The structure and formation of the Galactic bulge

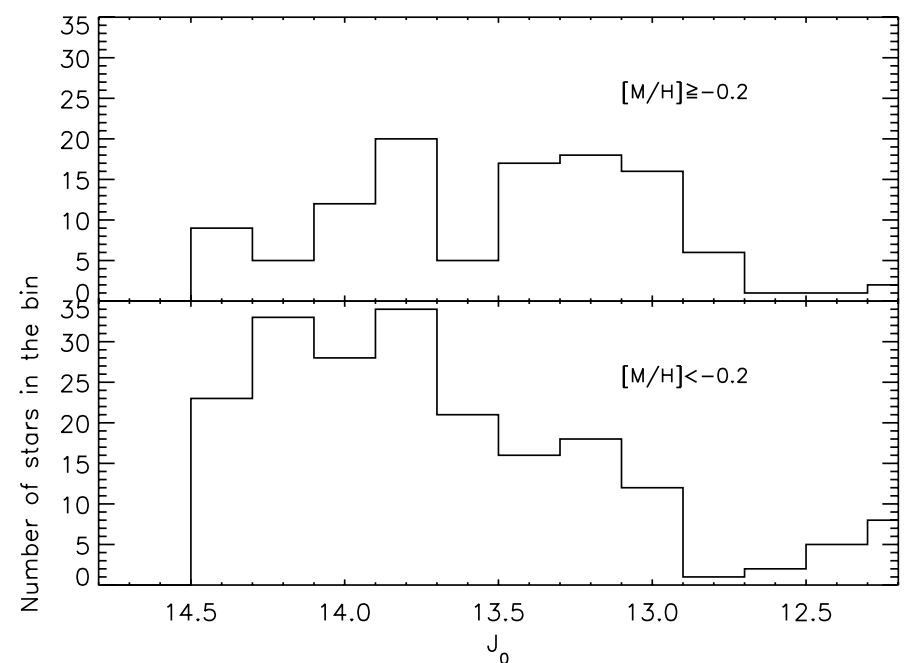

Fig. 10. Luminosity functions of the metal-rich ([M/H] $\geq-0.2$, upper panel) and the metal-poor $([\mathrm{M} / \mathrm{H}]<-0.2$, lower panel $)$ populations around the red clump brightness.

at brighter magnitudes, there are preferentially redder stars selected from the bright $\mathrm{RC}$, and bluer stars from the faint $\mathrm{RC}$ (see also Fig. 5). At a given evolutionary state, redder stars are more metal-rich than bluer ones. Hence, we selected preferentially more metal-rich stars in the bright RC than in the faint RC. This interpretation is also favoured by the RV distributions of the two RCs, which are practically indistinguishable (cf. Sect. 4.6). Introducing an additional colour criterion does not strengthen this conclusion because of the small number of stars that would be compared then.

In fact, we suggest that the double RC feature is connected only to the metal-rich population. A direct hint may come from the luminosity functions of the metal-poor and metal-rich populations around the RC brightness, see Fig. 10. The metallicity cut was introduced at $[\mathrm{M} / \mathrm{H}]=-0.2$ for this diagram. The metal-rich population has two peaks in the $J_{0}$ histogram, one at $J_{0} \sim 13.2$ and one at $J_{0} \sim 13$. 9 . It stands to reason that these two peaks are the manifestation of the front and back arms of the $\mathrm{X}$-shaped bulge. The fainter peak contains fewer stars because its stars are at larger distance from the Galactic plane where the density of stars is lower (Saito et al. 2011). The metal-poor population has one very broad, symmetric peak at $J_{0} \sim 14$ m 0 with a decline towards brighter magnitudes. Because the RC and the RGB bump have the same magnitude in $J_{0}$ at a metallicity of $[\mathrm{M} / \mathrm{H}] \approx-0.7$ (Nataf et al. 2011), this peak may also contain some RGB bump stars of the metal-poor population. A statistical t-test shows that the probability that the two luminosity functions are drawn from the same parent distribution is only $5.1 \%$. Also Ness et al. (2012), from an analysis of spectra of clump stars along the minor bulge axis, find that a split in the luminosity function appears only for stars with $[\mathrm{Fe} / \mathrm{H}]>-0.5$, in good agreement with our finding.

Another argument for the suggestion that the double RC feature is connected only to the metal-rich population may come from the analogy of how the double RC feature diminishes with increasing distance from the Galactic plane and the presence of a metallicity gradient in the bulge. CMDs based on 2MASS photometry in three different fields along the minor axis of the bulge (at $b=-7.0^{\circ},-8.5^{\circ}$, and $-10.0^{\circ}$, from top to bottom) are shown in Fig. 11. Fields with radii of $20^{\prime}, 30^{\prime}$, and $40^{\prime}$, respectively, were used to keep the total number of stars approximately constant. On the right hand side of each panel, a magnitude
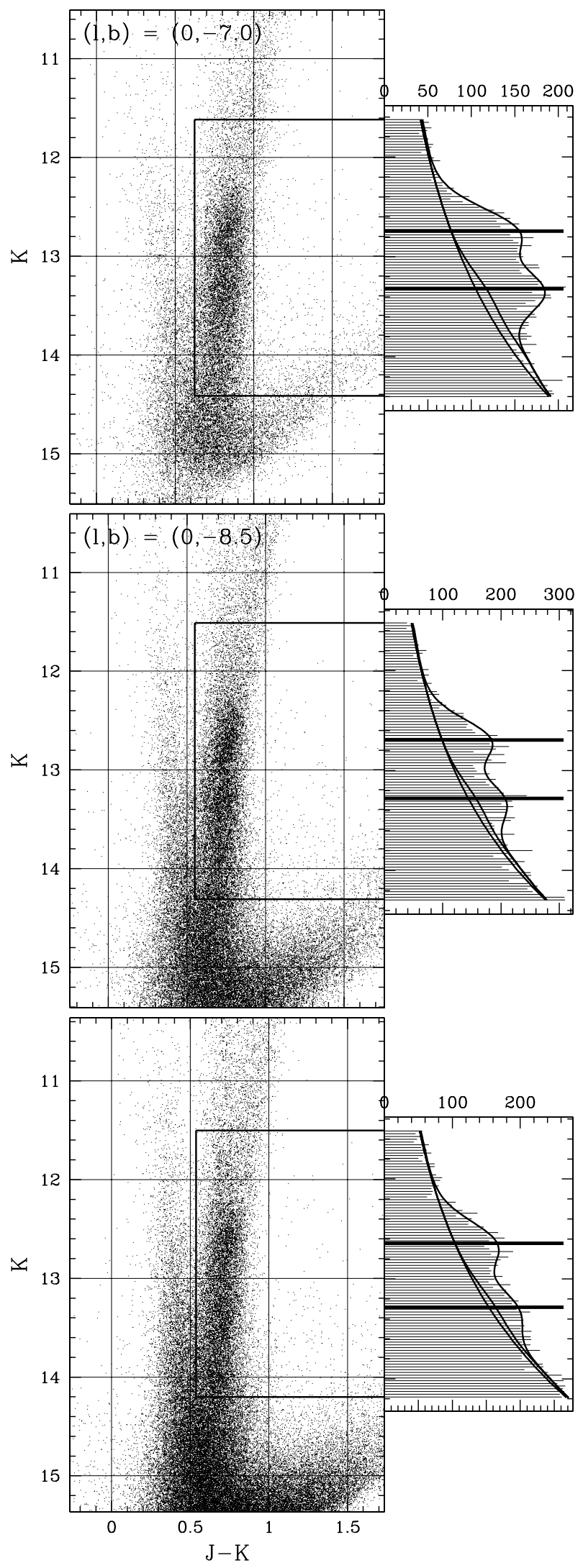

Fig. 11. Colour-magnitude diagrams of fields at $(l, b)=\left(0^{\circ},-7.0^{\circ}\right)$ (top panel $),(l, b)=\left(0^{\circ},-8.5^{\circ}\right)($ middle panel $)$, and $(l, b)=\left(0^{\circ},-10.0^{\circ}\right)$ (bottom panel). For each field, a magnitude histogram of the stars in the thick box in the CMD is shown in the right hand panel, including a fit to the double RC, the RGB bump, and the underlying distribution of RGB stars. The thick horizontal bars in the magnitude histograms mark the peak magnitudes of the two RCs. 


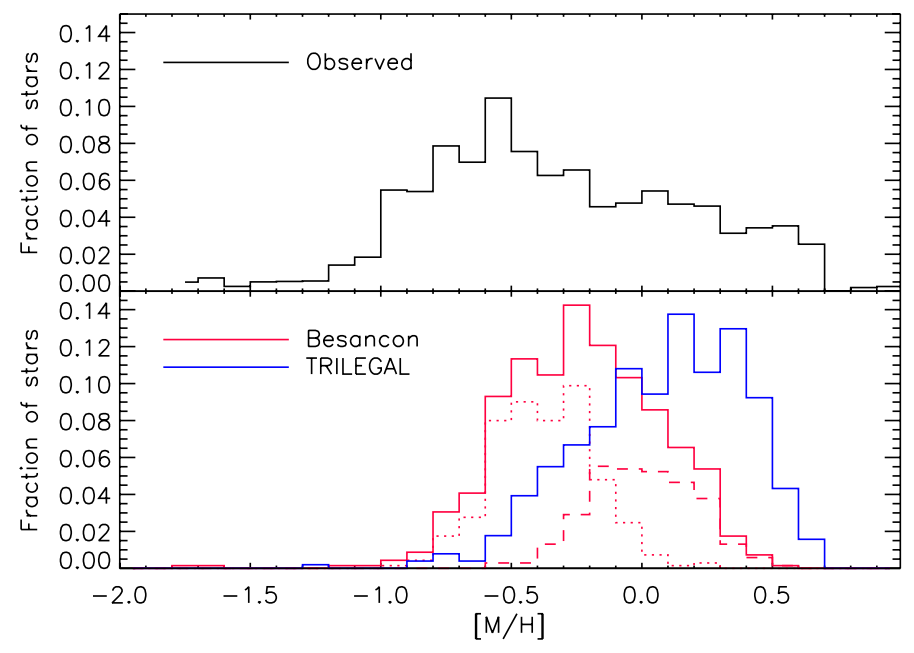

Fig. 12. Comparison of the observed MDF corrected for sampling effects (upper panel), with the ones predicted by the Besançon and TRILEGAL models in the selection region (lower panel). The histograms are normalised to the same area. In the lower panel, the red dotted line shows the MDF of "thick bulge" stars in the Besançon model, whereas the dashed red line is the one for the bar component.

histogram of the stars inside the box marked by thick lines is shown. The luminosity function was fitted with Gaussian and exponential functions to reproduce the RCs, RGB bump, and the underlying distribution of RGB stars. It is clear from that figure that not only the peak brightness positions of the RCs are moving apart when going away from the plane, but also the relative number of stars making up the double RC feature is decreasing with distance from the plane.

Together with the presence of a metallicity gradient in the bulge (Zoccali et al. 2008), this suggests that the double RC feature is connected only to the metal-rich population, which is much more concentrated towards the Galactic plane. In both Hill et al. (2011) and Bensby et al. (2011) the stars evenly distribute among the two sub-populations. In our sample, the metalrich population makes up only $\sim 30 \%$ of the stars (upper limit), which would suggest that the fraction of the populations varies with Galactic latitude. Hence, the metal-rich population seems to be more concentrated towards the plane than the metal-poor one. This would also explain the metallicity gradient found by Zoccali et al. (2008): if the fraction of metal-rich stars decreases with increasing distance from the plane, also the mean metallicity would shift to lower values. Hence, rather than a decreasing mean metallicity within one monolithic bulge population, probably it is the varying percentage of two sub-populations that mimics a metallicity gradient in the bulge.

\subsection{Comparison with simulated MDFs}

Our observed MDF, corrected for sampling effects (Sect. 4.1.1), is compared to the simulated MDFs from the Besançon and TRILEGAL models in Fig. 12. The simulated MDFs do not reproduce the observed MDF in all aspects. The MDF of the TRILEGAL model has clearly a much higher mean metallicity than the observed mean $([\mathrm{M} / \mathrm{H}]=+0.09$, compared to -0.34). The number of metal-poor stars is underestimated, whereas the super-solar metallicity stars are overrepresented in the TRILEGAL model. Clearly, the shift of +0.3 dex applied by Vanhollebeke et al. (2009) to the MDF of Zoccali et al. (2003) does not reproduce the MDF of our sample (which is at $-10^{\circ}$

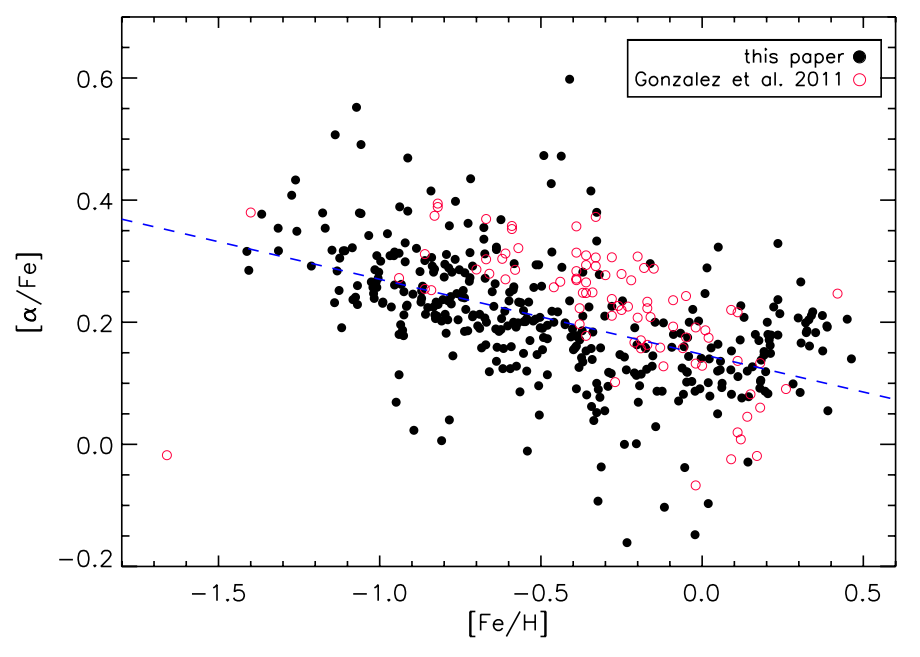

Fig. 13. Abundance of $[\alpha / \mathrm{Fe}]$ as a function of $[\mathrm{Fe} / \mathrm{H}]$. Filled black circles: data from this study; open red circles: data of the field at $b=-12^{\circ}$ from Gonzalez et al. (2011a). The dashed line is a linear fit trough the data of this paper.

from the Galactic plane). This probably means that an MDF calibrated on fields at low Galactic latitude cannot be simply applied to higher latitudes because of the metallicity gradient. The mean metallicity in the Besançon model, on the other hand, is close to the observed one $([\mathrm{M} / \mathrm{H}]=-0.23)$. For this model, the distributions of the bar (red dashed line) and the thick bulge (red dotted line) are shown separately in the lower panel of Fig. 12. The bar stars reproduce the metal-rich peak of the observed MDF relatively well. However, the two components are not separated in metallicity as much as they are in the observations. If the bulge component in the Besançon model was shifted to lower metallicities by 0.2 to $0.3 \mathrm{dex}$, it would very nicely reproduce the metal-poor peak in the observed MDF (cf. Sect. 4.7.1). We remind that these stellar population synthesis models assume certain metallicity distributions for each Galactic component. Self-consistent chemical evolution models such as Ballero et al. (2007) are needed to do a detailed comparison.

\section{5. $\alpha$-element abundances}

An important piece of evidence for the understanding of the GB population is the abundance of the $\alpha$-elements because they convey information of the star formation history. In Fig. 13 we present the abundance of $[\alpha / \mathrm{Fe}]$ as a function of the iron abundance $[\mathrm{Fe} / \mathrm{H}]$. The metal-rich and metal-poor stars also differ in this diagram: the metal-rich stars have clearly a lower overabundance in the $\alpha$-elements than the metal-poor ones. Among the metal-rich stars, most stars fall in the range $[\alpha / \mathrm{Fe}]=+0.05$ to +0.25 , while among the metal-poor stars many stars can be found at over-abundances of $+0.30 \mathrm{dex}$ and more.

Because of proximity to our field, we over-plot the abundances measured by Gonzalez et al. (2011a) in the field at $b=-12^{\circ}$. To convert the individual abundances to a combined $[\alpha / \mathrm{Fe}]$ value, we computed the weighted mean of their $\mathrm{Ca}, \mathrm{Ti}$, $\mathrm{Si}$, and $\mathrm{Mg}$ abundances, using as weight the contribution to the absorption in the synthetic spectrum that we applied in the determination of the $\alpha$-abundances (Sect. 3.1.3). The match between our data and those of Gonzalez et al. (2011a) is not perfect. The over-abundances measured by Gonzalez et al. (2011a) are on average slightly higher than in our data, even though a shift was applied to our data to take into account a systematic offset 


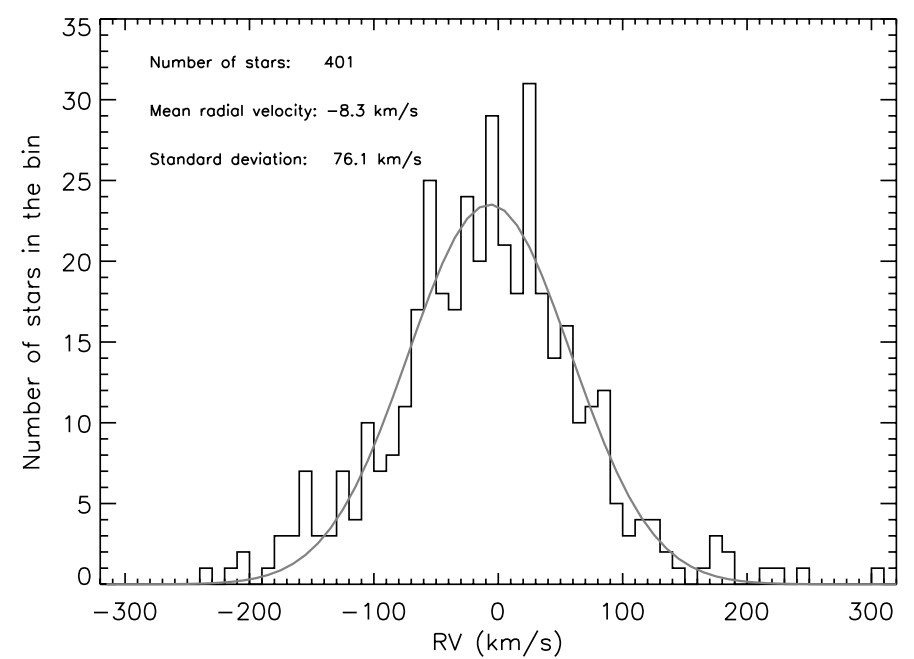

Fig. 14. Radial velocity distribution of our sample stars. The grey graph is a Gaussian fit to the data with mean and standard deviation as indicated in the figure.

(Sect. 3.1.4). It is also clear that there are many more metalpoor stars $(-1.5 \leq[\mathrm{Fe} / \mathrm{H}] \leq-0.5)$ in our sample than in that of Gonzalez et al. (2011a), and also at the metal-rich end we find somewhat more stars than they do.

We also confirm the trend of decreasing $\alpha$-element overabundance with increasing metallicity, as was found already by a number of previous studies (e.g. Fulbright et al. 2007; Ryde et al. 2010; Alves-Brito et al. 2010; Hill et al. 2011; Gonzalez et al. 2011a; Johnson et al. 2011a; Bensby et al. 2010a). A linear regression including all sample stars yields a slope of -0.12 (dashed line in Fig. 13). The $1 \sigma$ scatter around this slope is only $\sim 0.08 \mathrm{dex}$, which suggests that it originates only from measurement error, not from real abundance scatter within the sample, at a given metallicity. At the high metallicity end, the trend of decreasing $\alpha$-element over-abundance might be shallower or even reversed. This seems to disagree with the data of Gonzalez et al. (2011a) overplotted in Fig. 13, however. On the other hand, a flat trend with $[\mathrm{Fe} / \mathrm{H}]$ is also found in metal-rich, microlensed bulge dwarf stars for some $\alpha$-elements, e.g. $\mathrm{Ca}, \mathrm{Ti}, \mathrm{Si}$, and $\mathrm{Mg}$ (Fig. 10 of Bensby et al. 2011). Only oxygen is clearly found to continue its decrease at high metallicity. If this is a real feature or the result of uncertainties needs to be investigated with more precise line-by-line studies and larger samples, respectively.

\subsection{Radial velocities}

The RV distribution is an invaluable constraint on the nature of the GB, i.e. to decide whether it is a classical or a pseudobulge. The mean RV of our whole sample is $-8.3 \pm 3.8 \mathrm{~km} \mathrm{~s}^{-1}$, with a velocity dispersion $\sigma_{\mathrm{RV}}=76.1 \pm 2.7 \mathrm{~km} \mathrm{~s}^{-1}$. (Note: in the following, the quoted uncertainties in the mean velocity and the standard deviation of the distribution are always the purely statistical uncertainties.) The distribution of RVs of our sample stars is displayed in Fig. 14. No cold streams are obvious in our sample. Excluding the 50 foreground candidates identified in Sect. 2.3 by their high proper motion (and also the 10 stars for which no proper motion measurement was found), we get $\langle R V\rangle=-8.9 \pm 4.2 \mathrm{~km} \mathrm{~s}^{-1}$ and $\sigma_{\mathrm{RV}}=77.1 \pm 3.0 \mathrm{~km} \mathrm{~s}^{-1}$, hence no significant change.

We can compare our observed $\sigma_{\mathrm{RV}}$ to those of Babusiaux et al. (2010) and the BRAVA team (Howard et al. 2008, 2009). The comparison is done in Fig. 15, which is a reproduction of

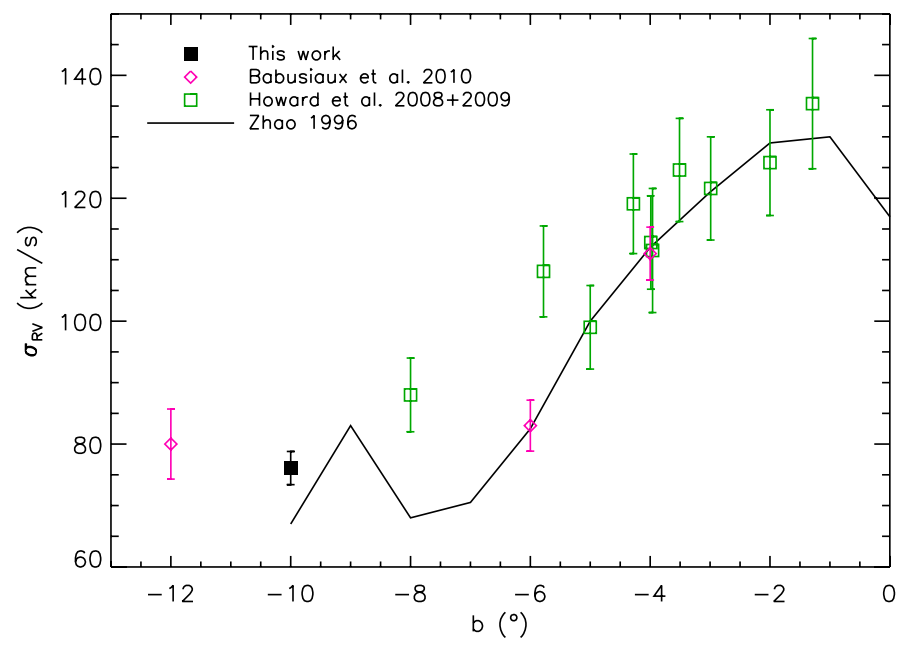

Fig. 15. Comparison of observed velocity dispersions as a function of Galactic latitude $b$ along the bulge minor axis $\left(l=0^{\circ}\right)$ with the model of Zhao (1996). Cf. Fig. 7 of Babusiaux et al. (2010).

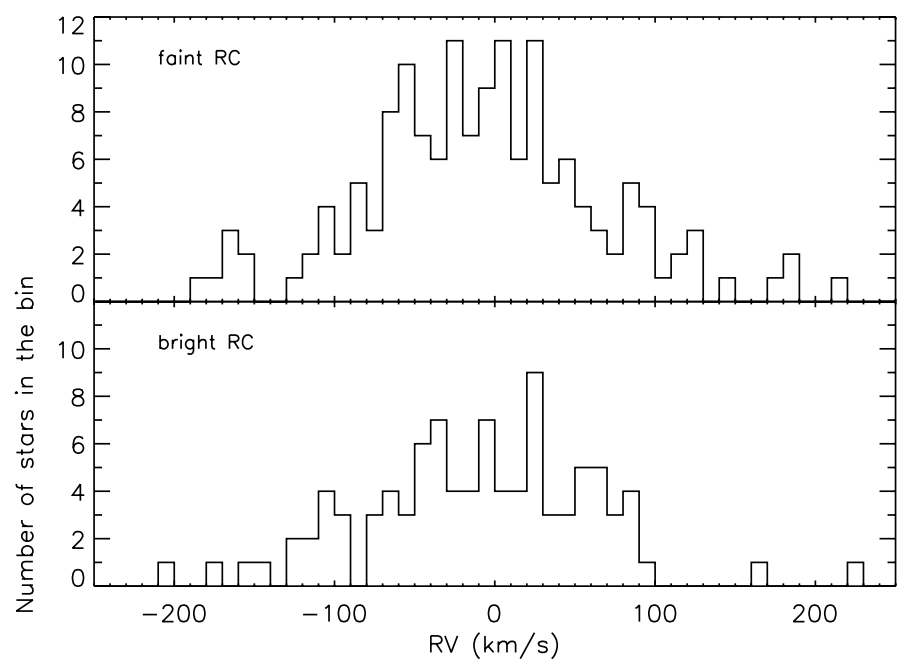

Fig. 16. Radial velocity distribution of the stars in the bright and faint $\mathrm{RC}$, respectively.

Fig. 7 of Babusiaux et al. (2010). We emphasise that the sample selection for the BRAVA survey, Babusiaux et al. (2010), and for the present study are quite different. From the BRAVA survey, only the results for fields along the bulge minor axis $\left(l \approx 0^{\circ}\right)$ are shown. Our measurement of $\sigma_{\mathrm{RV}}$ is the lowest one in this figure, but within the error bar of the data point at $b=-12^{\circ}$ from Babusiaux et al. (2010). Figure 15 also includes the prediction from the model by Zhao (1996), which is a "3D steady-state stellar dynamic model for the Galactic bar", hence a pseudo-bulge model. The observed $\sigma_{\mathrm{RV}}$ as a function of $b$ is well described by the model of Zhao (1996), except for the two BRAVA data points farthest from the Galactic plane. The $\sigma_{\mathrm{RV}}$ of our sample is only slightly higher than what is predicted by this model. Furthermore, our measurement is also in excellent agreement with the predictions of the pseudo-bulge model developed by Shen et al. (2010, their Fig. 2, lower right panel).

The kinematics of the RC stars is an important test for the understanding of the nature of the double RC feature. Figure 16 displays the RV distribution of the stars in the two RCs as defined in Sect. 2.2. The mean RVs are $-10.4 \pm 7.3$ and $-6.0 \pm 6.1 \mathrm{~km} \mathrm{~s}^{-1}$, and the standard deviations are $71.6 \pm 5.2$ and $74.1 \pm 4.3 \mathrm{~km} \mathrm{~s}^{-1}$, respectively. Although the velocity dispersion of the faint $\mathrm{RC}$ is 
Table 2. Radial velocity distributions of different sub-samples.

\begin{tabular}{lccc}
\hline \hline Selection & $N$ & $\langle R V\rangle\left(\mathrm{km} \mathrm{s}^{-1}\right)$ & $\sigma_{\mathrm{RV}}\left(\mathrm{km} \mathrm{s}^{-1}\right)$ \\
\hline Observations & & & \\
whole sample & 401 & $-8.3 \pm 3.8$ & $76.1 \pm 2.7$ \\
$\mu<20$ mas & 341 & $-8.9 \pm 4.2$ & $77.1 \pm 3.0$ \\
bright RC & 96 & $-10.4 \pm 7.3$ & $71.6 \pm 5.2$ \\
bright RC, weighted & 96 & $-10.3 \pm 7.3$ & $71.3 \pm 5.2$ \\
faint RC & 150 & $-6.0 \pm 6.1$ & $74.1 \pm 4.3$ \\
faint RC, weighted & 150 & $-5.1 \pm 6.1$ & $73.5 \pm 4.1$ \\
metal-rich third & 128 & $-7.1 \pm 4.6$ & $52.3 \pm 3.3$ \\
metal-poor third & 128 & $-5.4 \pm 8.0$ & $90.6 \pm 5.7$ \\
metal-rich pop. ${ }^{1}$ & 88 & $-6.1 \pm 5.3$ & $47.7 \pm 5.6$ \\
metal-poor pop. ${ }^{1}$ & 198 & $-9.1 \pm 6.4$ & $90.3 \pm 6.5$ \\
\hline Besançon model & & & \\
whole sample & 6933 & $-11.1 \pm 0.9$ & $77.8 \pm 0.7$ \\
$\mu<20$ mas & 6555 & $-11.0 \pm 1.0$ & $79.6 \pm 0.7$ \\
bright RC & 1408 & $-10.0 \pm 2.0$ & $74.1 \pm 1.4$ \\
faint RC & 2802 & $-11.1 \pm 1.5$ & $80.9 \pm 1.1$ \\
metal-rich third & 2311 & $-13.1 \pm 1.4$ & $65.9 \pm 1.0$ \\
metal-poor third & 2312 & $-9.2 \pm 1.8$ & $87.2 \pm 1.3$ \\
\hline
\end{tabular}

Notes. The top group are the values measured from the observed sample, the bottom group are the values derived from ten simulation runs of the Besançon model (Robin et al. 2012). ${ }^{(1)}$ Metal-rich and metal-poor population according to the Gaussian decomposition in Sect. 4.1.2.

slightly larger than that of the bright RC, the two distributions are indistinguishable, within the error bars. To make this result more significant, we derived probabilities that a particular star belongs to either the front or back over-density (bright or faint $\mathrm{RC}$ ). For this end we fitted two Gaussians (for the two RCs) and an exponential function (for the underlying RGB stars) to the magnitude histogram to derive these probabilities. This procedure was applied to a 40' diameter field to increase the statistics for a Gaussian fitting of the two RCs. Using these membership probabilities as weights, we get $\langle R V\rangle=-10.3 \pm 7.3 \mathrm{~km} \mathrm{~s}^{-1}$ and $\langle R V\rangle=-5.1 \pm 6.0 \mathrm{~km} \mathrm{~s}^{-1}$, and $\sigma_{\mathrm{RV}}=71.3 \pm 5.2 \mathrm{~km} \mathrm{~s}^{-1}$ and $\sigma_{\mathrm{RV}}=73.5 \pm 4.1 \mathrm{~km} \mathrm{~s}^{-1}$ for the bright and faint $\mathrm{RC}$, respectively. Even in the weighted mean, the kinematics of the two RCs remain indistinguishable. Furthermore, a statistical t-test yields a probability of $64.2 \%$ that the two distributions are drawn from the same parent population.

The above discussed mean RVs and velocity dispersions of the different sub-samples are summarised in Table 2.

\subsection{Combining kinematics with abundances}

The upper panel of Fig. 17 shows how the sample stars distribute in the RV vs. $[\mathrm{M} / \mathrm{H}]$ plane. It it immediately clear from this diagram that the RV distribution becomes broader with decreasing metallicity and that at low metallicity more extreme RV values are found than at high metallicity. This is also illustrated by the large over-plotted "error-bar" symbols, which show the mean RV and velocity dispersion over the metallicity range between the vertical dotted lines. The mean RV stays more or less constant, whereas $\sigma_{\mathrm{RV}}$ increases monotonically with decreasing metallicity. This is further evidence that there are two different populations present in our sample, each with its own velocity distribution. Whereas the increase in the velocity dispersion agrees with the results of de Propris et al. (2011), the lack of a trend of the mean RV with metallicity is in clear contrast to their results. This also holds if only the RC stars in our sample are considered. This is somewhat surprising because the field studied by de Propris et al. (2011) is only two degrees closer to the Galactic

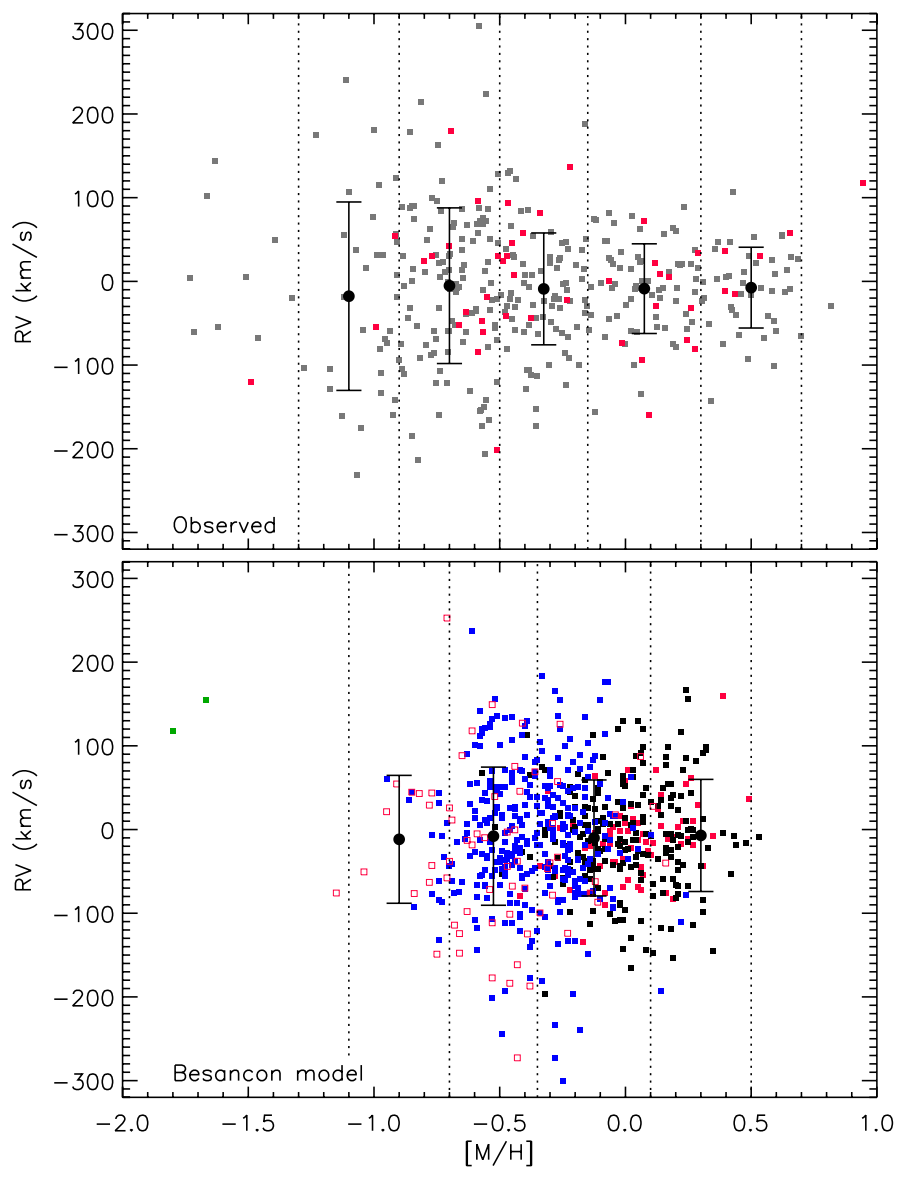

Fig. 17. Radial velocity versus metallicity. Top panel: observed sample stars. Foreground star candidates are represented by red squares, all other stars by grey squares. Bottom panel: simulated stars in the Besançon model (Robin et al. 2012). Blue squares represent the thick bulge, black squares the bar, filled red squares the thin disc, open red squares the thick disc, and green symbols represent the halo population. The over-plotted error-bar symbols in both panels show the mean and standard deviation of the RV over the metallicity range between the vertical dotted lines.

plane than our field, along the bulge minor axis. At this point we do not have an explanation for this discrepancy.

The metal-rich stars on the near side of the X (stars in the bright peak in the upper panel of Fig. 10) may be expected to differ in their mean radial velocity from those on the far side (faint peak in Fig. 10). We find $\langle R V\rangle=+1.5 \pm 7.5 \mathrm{~km} \mathrm{~s}^{-1}$ and $\sigma_{\mathrm{RV}}=53.4 \pm 5.3 \mathrm{~km} \mathrm{~s}^{-1}$ for the 51 bright, metal-rich $\mathrm{RC}$ stars and $\langle R V\rangle=-10.7 \pm 8.2 \mathrm{~km} \mathrm{~s}^{-1}$ and $\sigma_{\mathrm{RV}}=51.5 \pm 5.9 \mathrm{~km} \mathrm{~s}^{-1}$ for the 39 faint, metal-rich RC stars, respectively (using a metallicity cut at $[\mathrm{M} / \mathrm{H}] \geq-0.2$ and the brightness criteria for the RCs of Sect. 2.2). The error bars on the mean RVs overlap, hence the difference is not significant. Note, however, that RGB/early AGB stars brighter than the RC located on the far side and stars fainter than the RC located on the near side will dilute any difference between these two groups. A still larger sample would be needed to confirm this difference in mean RV.

\subsubsection{Comparison with the Besançon model}

It is interesting to compare this result with the kinematics as provided by the simulations to see whether the BGM, based on a decomposition in several populations, mainly disc, bulge and bar in the central region, is in reasonable agreement with our data. The 


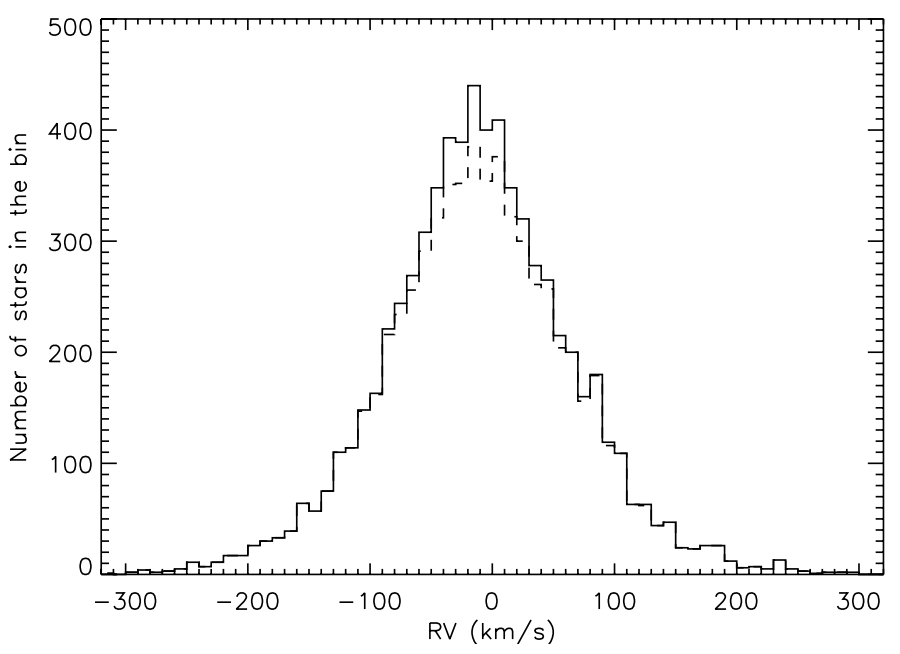

Fig. 18. Radial velocity distribution of stars in ten simulation runs of the Besançon model (Robin et al. 2012) for our field. The full line is for all stars in the selection region, the dashed line shows the distribution for stars with total proper motion $\mu<20$ mas/yr.

lower panel of Fig. 17 shows for comparison the distribution of simulated stars in the BGM in the RV vs. metallicity plane. The width of the RV distribution as well as the extreme RV values agree well with those in the observed sample. However, the trend of increasing velocity dispersion with decreasing metallicity is much smaller in the simulation than in the observations. The main reason for this is that the bulge population (blue symbols in the lower panel of Fig. 17), whose velocity dispersion is relatively large, is close in metallicity to the bar population (black symbols), which has a smaller velocity dispersion. If the bulge population in the Besançon model was shifted to lower metallicities by 0.2 to $0.3 \mathrm{dex}$, it would nicely reproduce this trend, and also bring the range of metallicities in the model closer to the observed range. This is in line with the conclusions drawn from the comparison of the MDFs (Sect. 4.4).

To compare the observed with the simulated velocity dispersions, and to decrease the Poisson noise in the model, we calculated ten simulation runs of the Besançon model for our field. From these ten simulations, we obtain 6933 stars in the selection area. The results of this exercise are summarised in the lower part of Table 2. The mean RV as well as the velocity dispersion of the simulation agree well with the observed values for the whole sample. Excluding stars with a total proper motion $\mu>20 \mathrm{mas} / \mathrm{yr}$, the velocity dispersion slightly increases in both observations and simulations. As discussed in Sect. 3.2.3, this selection by proper motion is very efficient in removing the foreground disc. This increase in velocity dispersion is not significant in the observed sample, but it is significant in the simulation. In Fig. 18 we show the histogram of the distribution of radial velocity from the simulations, with (dashed line) and without (solid line) the proper motion selection. As can be seen from this figure, the proper motion selection removes a cold (disc) component.

Table 2 also lists the mean RV and dispersions of the simulated bright and faint red clump sub-samples, as well as for the metal-rich and metal-poor $33.3 \%$ quantile. In both the observations and in the simulations the bright RC has a slightly smaller velocity dispersion; while this increase is again not significant in the observed sample, it is significant in the simulations. The reason for this probably is that, as already discussed in Sect. 4.3, the faint RC is more contaminated by stars from the metal-poor population, which has a higher velocity dispersion than the bar population, the probable carrier of the double clump feature.

The metal-rich sample has a smaller velocity dispersion than the metal-poor one. This is due to the fact that the metal-rich one is dominated by the bar population, while the more metalpoor one is dominated by the bulge. The velocity dispersion of the metal-poor third of the simulation agrees nicely with the observed one $\left(87.2 \mathrm{~km} \mathrm{~s}^{-1}\right.$ to be compared with $90.6 \mathrm{~km} \mathrm{~s}^{-1}$, see below). However, the metal-rich third has a larger dispersion in the simulations $\left(65.9 \mathrm{~km} \mathrm{~s}^{-1}\right)$ than in the observed sample $\left(52.3 \mathrm{~km} \mathrm{~s}^{-1}\right)$. As seen in the lower panel of Fig. 17, the metalrich part of the sample is dominated by the bar, which follows Fux (1999) dynamics. But there is also a contribution from the disc, which is mainly removed using the proper motion selection. In the very low metallicity tail, there are a few halo stars and a noticeable contribution from the thick disc. It has, in our model, a smaller dispersion than the bulge, which has about the same metallicity. The velocity dispersion of the thick disc in the inner galaxy is not known and the simulation we are proposing here is just an attempt to extrapolate the thick disc as seen in the solar neighbourhood to the central region. Though, the dispersion we obtain for this intermediate and low metallicity bin is in very good agreement with the data.

We conclude that this new model, having both a flaring bar and a bulge component in the central region, explains very well the observed relation between the metallicity and the kinematics in this bulge field.

\subsubsection{Comparison with the Fux model}

The increase of $\sigma_{\mathrm{RV}}$ with decreasing metallicity is reminiscent of the results of Babusiaux et al. (2010), who also found a clear distinction in kinematics between the metal-poor and the metalrich stars in bulge fields at different Galactic latitudes. In a reproduction of their Fig. 8, we show in Fig. 19 a comparison with the bulge dynamics as predicted by the $3 \mathrm{D}$ self-consistent $N$-body barred models of the Milky Way by Fux (1999). As in Babusiaux et al. (2010), $\sigma_{\mathrm{RV}}$ is plotted in different symbols for the complete sample (diamonds), the $33.3 \%$ metal-poor quantile (open downward-pointing triangles), and the $33.3 \%$ metal-rich quantile (filled upward-pointing triangles). We find $\sigma_{\mathrm{RV}}=52.3 \pm 3.3 \mathrm{~km} \mathrm{~s}^{-1}$ for the metal-rich third of the sample and $\sigma_{\mathrm{RV}}=90.6 \pm 5.7 \mathrm{~km} \mathrm{~s}^{-1}$ for the metal-poor third (Table 2). By adding only one more data point at $b=-10^{\circ}$ we can put much more weight on the conclusions of Babusiaux et al. (2010). Within the error bars, $\sigma_{\mathrm{RV}}$ of the metal-poor quantile is constant throughout the bulge, from $b=-4^{\circ}$ to $-12^{\circ}$. The spheroid model of Fux (1999) also predicts $\sigma_{\mathrm{RV}}$ to be fairly constant as a function of $b$, except for a dip around $b=-9^{\circ}$. However, the observed $\sigma_{\mathrm{RV}}$ is systematically lower than the $\sigma_{\mathrm{RV}}$ predicted by the spheroid model. The velocity dispersion of the $33.3 \%$ metalrich quantile, on the other hand, decreases monotonically with increasing distance from the Galactic plane. This follows closely the prediction by the disc/bar model of Fux (1999), but except at $b=-4^{\circ}$ it is also always below the predicted value.

We must caution at this point that, while the definition of quantiles is a robust measure, the metallicity cuts will be at different absolute values, depending on the metallicity scale used in a given study and on the metallicity distribution in the different fields. We therefore also derived the RV dispersions of the two populations from the Gaussian decomposition done in Sect. 4.1.2. Assuming that both have Gaussian RV distributions, we find that the metal-poor population has $\langle R V\rangle=-9.1 \pm 6.4 \mathrm{~km} \mathrm{~s}^{-1}$ with $\sigma_{\mathrm{RV}}=90.3 \pm 6.5 \mathrm{~km} \mathrm{~s}^{-1}$, while 


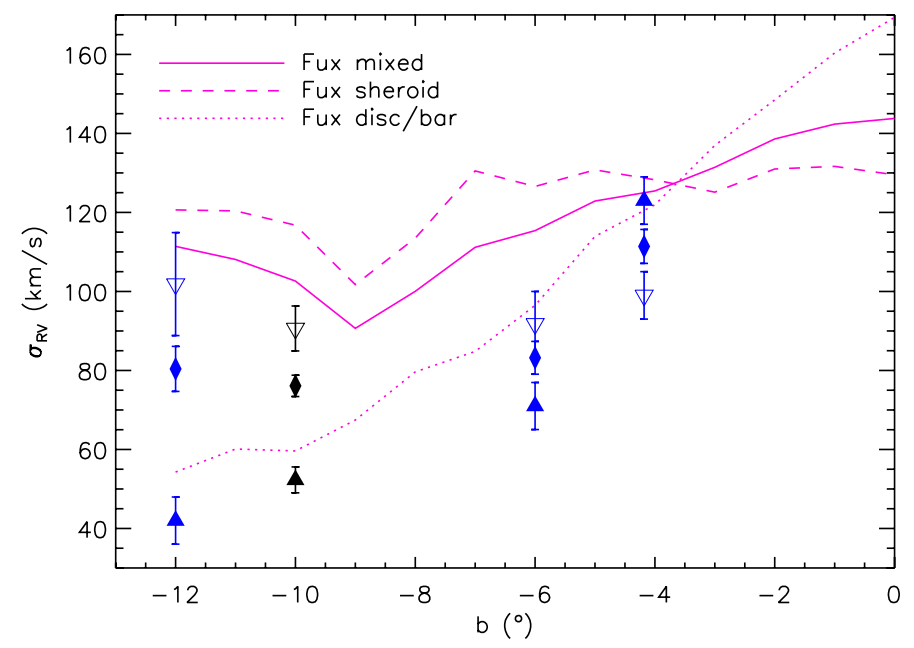

Fig. 19. Observed RV dispersions as a function of Galactic latitude $b$ along the bulge minor axis, compared to the models of Fux (1999), cf. Fig. 8 of Babusiaux et al. (2010). The blue symbols are the data points from Babusiaux et al. (2010), the black symbols are from this work. The diamond symbol represents the velocity dispersion of the complete sample, while the open downward-pointing triangle represents the $33.3 \%$ metal-poor quantile and the filled upward-pointing triangle represents the $33.3 \%$ metal-rich quantile, respectively.

the metal-rich population has $\langle R V\rangle=-6.1 \pm 5.3 \mathrm{~km} \mathrm{~s}^{-1}$ and $\sigma_{\mathrm{RV}}=47.7 \pm 5.6 \mathrm{~km} \mathrm{~s}^{-1}$ (Table 2$)$.

\subsubsection{Evidence for metallicity-dependent mass loss from the velocity dispersion of evolved stars}

In Sect. 4.2 we speculated that the lack of metal-rich stars among the bright sample stars might be caused by strong, metallicitydependent mass loss that preferentially removes metal-rich stars from the evolutionary pathes (the so-called AGB manqué). There is another, even stronger piece of evidence coming from the RV distributions of AGB stars and planetary nebulae (PNe) in the PG3 field that supports this explanation. RVs of Mira and semi-regular variables (SRVs) in the PG3 have been presented by Schultheis (1998) and Uttenthaler et al. (2007). The 27 AGB stars studied by Uttenthaler et al. (2007) by means of high-resolution UVES/VLT spectra have a velocity dispersion of $90.9 \pm 12.6 \mathrm{~km} \mathrm{~s}^{-1}$. Schultheis (1998) found $\sigma_{\mathrm{RV}}=$ $95.2 \pm 8.8 \mathrm{~km} \mathrm{~s}^{-1}$ for 59 PG3 Miras and $\sigma_{\mathrm{RV}}=83.0 \pm 6.1 \mathrm{~km} \mathrm{~s}^{-1}$ for 94 SRVs. If additional criteria based on the period $K$-magnitude relation are applied to select only probable bulge members, these numbers change to $\sigma_{\mathrm{RV}}=110.4 \pm 13.2 \mathrm{~km} \mathrm{~s}^{-1}$ (Miras, $N=36)$ and $\sigma_{\mathrm{RV}}=89.4 \pm 9.6 \mathrm{~km} \mathrm{~s}^{-1}(\mathrm{SRVs}, N=$ 43). Furthermore, we retrieved RV measurements of PNe from the compilation of Durand et al. (1998). Nineteen PNe were found within a search radius of $4^{\circ}$ from the centre of our FLAMES field. Those $19 \mathrm{PNe}$ have a velocity dispersion of $94.1 \pm 15.7 \mathrm{~km} \mathrm{~s}^{-1}$. Although this last number is not highly significant owing to the small number of objects, it is clear that these velocity dispersions agree very well with the one determined for the metal-poor sub-population, but disagree with that of the metal-rich one. This statement is summarised in Fig. 20. We conclude from this that the AGB stars and PNe in the PG3 field descend mainly from the metal-poor sub-population. Or, to say it the other way round, we conclude that the metal-rich stars probably never evolve up to the AGB stage and beyond because they terminate their evolution before. This conclusion has also

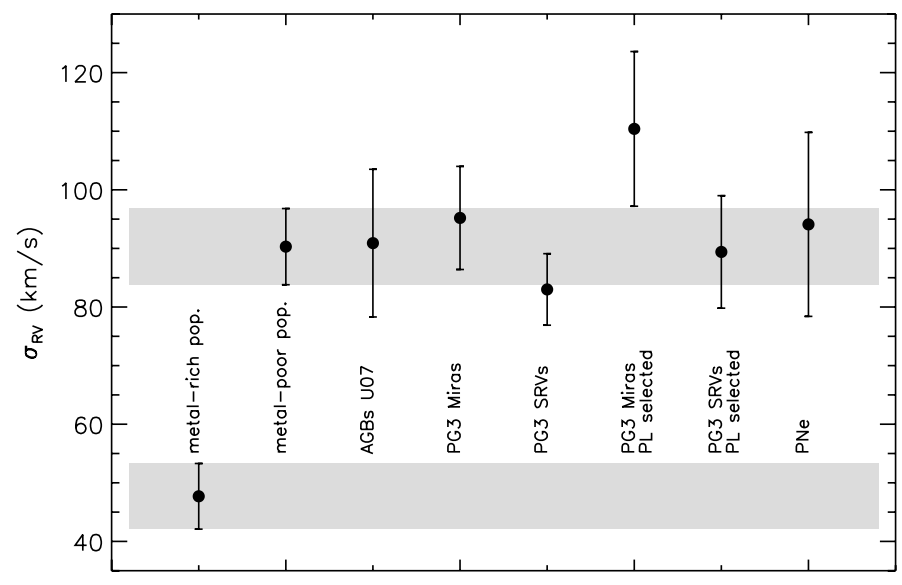

Fig. 20. Velocity dispersion of the metal-rich and metal-poor populations (Table 2), as well as of several samples of evolved stars towards the PG3 field: AGB stars from Uttenthaler et al. (2007, U07), Miras and SRVs from Schultheis (1998) without and with selection using a period - K-magnitude relation, and PNe from Durand et al. (1998). The grey shaded areas mark the range of uncertainty of the metal-rich and metal-poor population, respectively.

important consequences on the interpretation of the dual chemistry found in many bulge PNe (Guzman-Ramirez et al. 2011), a phenomenon which was previously assumed to be a consequence of high-metallicity PNe precursor stars (Perea-Calderón et al. 2009).

Apparently, there is a significant metal-rich population present out to $b \geq-10^{\circ}$, however it seems to be unable to produce highly evolved stars. Also the dearth of super-solar metallicity stars among $\mathrm{M}$ giants in fields close to the Galactic plane (Rich et al. 2007, 2012) may be caused by enhanced mass loss off metal-rich stars, in line with our result and interpretation. Finally, enhanced mass loss might also be required to understand the binary fraction of low-mass white dwarfs (Brown et al. 2011). A puzzle in this context is the presence of technetiumrich, long-period Mira stars towards the PG3 field (Uttenthaler et al. 2007), which suggest the presence of a younger and more massive $\left(M \approx 1.5 M_{\odot}\right)$ population in the $\mathrm{GB}$, but at the same time carbon stars are extremely rare or even absent in the bulge (Blanco \& Terndrup 1989; Ng 1997; Schultheis 1998). This seems to be incompatible with the hypothesis of bulge AGB stars descending only from the old, metal-poor population. A solution to this problem could be that the Tc-rich AGB stars are the result of blue straggler evolution. Also problematic in this respect are the various selection effects that are certainly present on the AGB and PN samples in the GB, and only more detailed investigations, e.g. on the metal content of bulge AGB stars, can give more reliable answers.

A natural consequence of the loss of metal-rich stars by enhanced stellar winds would be that samples of bright M-type giants, as used by the BRAVA survey to probe the bulge velocity dispersion, could be biased towards metal-poor stars. In Sect. 4.6 we found that the observed $\sigma_{\mathrm{RV}}$ as a function of $b$ is well described by the model of Zhao (1996), except for the two BRAVA data points farthest from the Galactic plane (Fig. 15). A bias towards low metallicity could be the reason why the $\sigma_{\mathrm{RV}}$ measured by BRAVA at large distances from the plane are systematically higher than the predictions by the Zhao (1996) model: at this Galactic latitude, the velocity dispersions of the metal-rich and the metal-poor populations differ considerably, thus a bias 

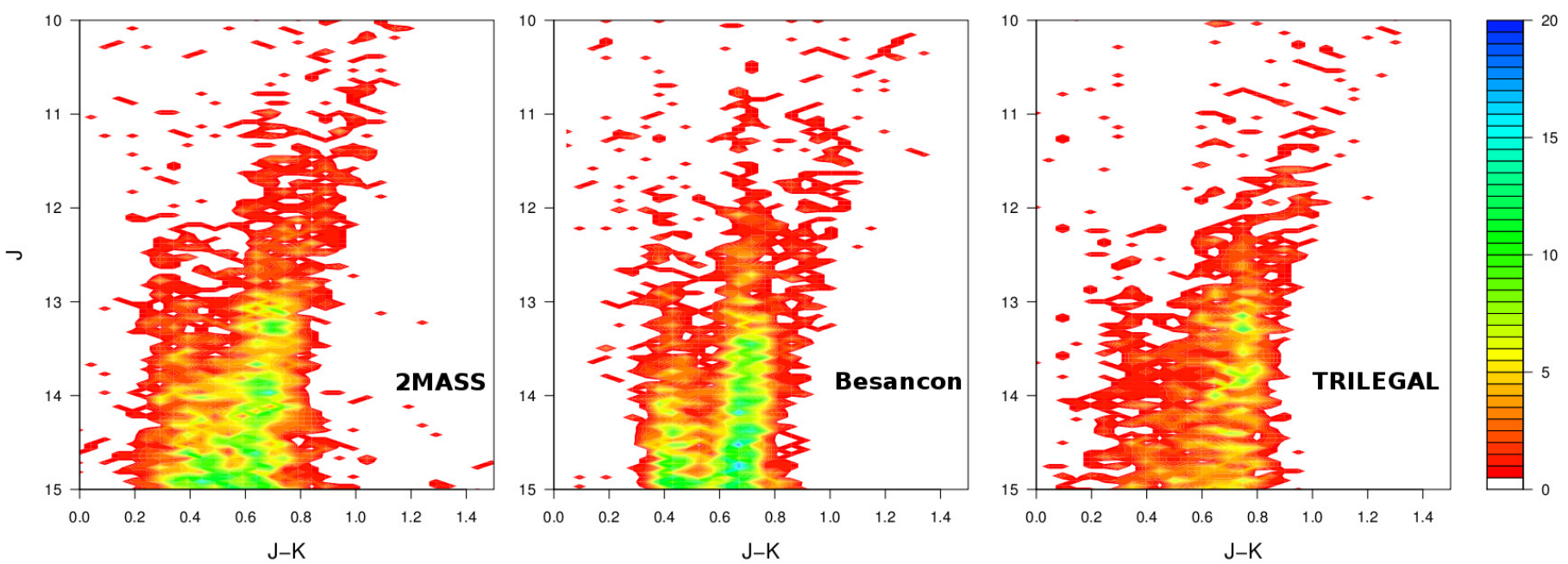

Fig. 21. Observed and simulated CMDs plotted as density maps. Left panel: observed CMD from 2MASS. Middle panel: simulated CMD from the Besançon model. Right panel: simulated CMD from the TRILEGAL model. The number of stars is not normalised. The scale on the right hand side illustrates to what colours the density of stars are connected in the diagrams.

towards more metal-poor stars will yield an enhanced velocity dispersion.

\section{Comparison of observed and simulated CMDs}

Comparisons with observed CMDs are an important test of Galaxy models. The 2MASS all-sky catalogue is an ideal source for such constraints (see e.g. Robin et al. 2012). In Fig. 21 we provide a comparison for our field. No extinction correction was applied to the observed CMD. Rather, we applied the Marshall et al. (2006) 3D extinction model to both the TRILEGAL and BGM simulations. In addition, photometric errors in each filter of the order of those in the 2MASS catalogue were added to both the Besançon and the TRILEGAL simulation.

The CMD in this area of the sky is dominated by three sequences: a sequence on the blue side formed by main-sequence disc stars in the foreground, a vertical sequence formed by red clump stars distributing at various distances from the sun, and a red sequence formed by bulge stars, along which our selection of spectroscopic targets was made. These three sequences are most clearly resolved in the Besancon model (middle panel of Fig. 21), even though photometric errors of the same order of magnitude as in the 2MASS catalogue have been added to the simulated stars.

The two red clumps in the observed CMD at $J \sim 13.3$ and $J \sim 14$. 0 are not well reproduced in the simulated CMDs for this field. This is not surprising for the TRILEGAL model, which uses a triaxial structure to describe the bulge. On the other hand, it has been shown that the flaring bar model developed by Robin et al. (2012) can reproduce the double clump feature, at least for a field at $(l, b)=\left(0^{\circ},-7^{\circ}\right)$. The double clump feature is not obvious in this single simulation plotted here, but an inspection of the ten combined simulations (Sect. 4.7.1), which were calculated to reduce the Poisson noise, the two clumps are well discernible at $J$ magnitudes of $\sim 13$. 6 and $\sim 14$. 1 . Hence, while the faint RC is well matched by the Besançon model, the bright one is slightly underestimated in its brightness (e.g. due to an overestimation of its distance).

In the TRILEGAL CMD a vertical sequence of RC stars is dominating in the bulge, which is probably a result of the triaxial bulge structure seen almost end-on, such that the bulge RC stars distribute over a relatively large range of distances from the sun.
Furthermore, the number of stars in the disc sequence seems to be lower in the TRILEGAL simulation than it is in both the observed and the Besançon CMD. The number of stars in the CMDs of Fig. 21 is not normalised; star counts constitute an important test of population synthesis models (Girardi et al. 2005). In the colour and magnitude range of Fig. 21, the observed CMD contains 2148 stars, while the Besançon and TRILEGAL models contain 2456 and 1476 stars, respectively.

While some details in the simulated CMDs somewhat deviate from observed CMDs, it is clear that the Galaxy models have reached already a very high level of accuracy and can reproduce most of the stellar populations at least qualitatively.

\section{Conclusions}

We presented an analysis of spectra of $\sim 400$ red giant stars towards a field at $(l, b)=\left(0^{\circ},-10^{\circ}\right)$. From these spectra, we derive metallicities, iron and $\alpha$-element abundances, and radial velocities. It is the first study that presents a homogeneous analysis of stars on the upper and lower RGB of the Galactic bulge. These data, as well as photometric data from the 2MASS survey, are compared to predictions by the TRILEGAL (Girardi et al. 2005) and Besançon (Robin et al. 2012) models of the Galaxy. These models are also used extensively to interpret our observations.

The mean metallicity of the whole sample is $[\mathrm{M} / \mathrm{H}]=$ -0.34 , and the radial velocity dispersion is $\sigma_{\mathrm{RV}} \sim 76 \mathrm{~km} \mathrm{~s}^{-1}$. In this study we confirm the presence of two sub-populations in the bulge at peak metallicities that differ by $\sim 0.9$ dex. The peak metallicities are found at $[\mathrm{M} / \mathrm{H}] \sim-0.6$ and $\sim+0.3$, with roughly equal dispersions. The sub-populations have significantly different kinematics and $\alpha$-element abundances: the metal-rich population has a narrow velocity distribution $\left(\sigma_{\mathrm{RV}} \sim\right.$ $50 \mathrm{~km} \mathrm{~s}^{-1}$ ) and low $\alpha$-abundances, whereas the metal-poor one has a broad distribution in radial velocities $\left(\sigma_{\mathrm{RV}} \sim 90 \mathrm{~km} \mathrm{~s}^{-1}\right)$ and high $\alpha$-abundances. The metal-rich population makes up $\sim 30 \%$ of our sample. This confirms and fosters recent results by Babusiaux et al. (2010), Bensby et al. (2011), and Hill et al. (2011) in most aspects. Also the kinematic models of Zhao (1996) and Fux (1999) are confronted with our data, and good agreement is found.

Furthermore, we find support for the suggestion by Babusiaux et al. (2010) that the ratio in number of these two 
sub-populations is a function of angular distance from the Galactic plane, which explains the observed metallicity gradient. The new scheme in the Besançon Galaxy model explains well the features seen in this sample, with two populations, a flaring metal-rich bar and a more metal-poor classical or thick bulge, even though the mean metallicity of this thick bulge should be adjusted downward by 0.2 to 0.3 dex. The metallicity distribution simulated with the TRILEGAL model yields a clearly too high mean metallicity to fit our observations.

This study also presents the first medium-resolution, high$\mathrm{S} / \mathrm{N}$ spectra of stars belonging to the two red clumps of the bulge. A small difference in mean metallicity between the two RCs is attributed to selection effects. We find that the double RC might be entirely due to the metal-rich sub-population. The kinematic difference between the two RCs when measured with radial velocities alone is at most very small. Some difference in the mean radial velocity of bright and faint metal-rich RC stars is found, although a still larger sample would be needed to confirm that stars on the far side preferentially move faster towards us than the ones on the near side.

We also find indications that the metallicity distribution function of the bulge might depend on the evolutionary state of the considered sample stars. In particular, there are fewer metalrich stars present among the brighter, more evolved stars in our sample. Although this result could be a consequence of selection bias or the still limited number of stars in our sample, we interpret this as the result of strong mass loss that causes the most metal-rich stars to terminate their evolution before the most advanced states. A very robust indication for that comes from the fact that AGB stars and PN in the outer bulge have a velocity dispersion that agrees with that of the metal-poor population identified here, but clearly disagrees with that of the metal-rich population. We conclude, hence, that metal-rich stars might lose so much mass that they skip phases of post-main-sequence stellar evolution. This means that the selection of $\mathbf{M}$ giants in the BRAVA survey could be biased towards metal-poor stars. We also confirm the trend of decreasing $\alpha$-element over-abundance with increasing iron abundance, as found in previous studies (e.g. Gonzalez et al. 2011a).

Our study also provides for an explanation how the existence of a dual bulge identified here and elsewhere can be reconciled with kinematic studies that conclude that the Milky Way bulge might be a pure pseudo-bulge (e.g. Shen et al. 2010). In our version of the Besançon model, the (thick or classical) bulge population has only $4 \%$ the mass of the bar, even when its contribution is significant when one moves away from the plane. Thus it is compatible with the result of Shen et al. (2010), which finds that the fraction of the classical bulge cannot be more than $8 \%$ of the disc mass. The pseudo-bulge dominates, but there is some space for a classical bulge, which we see in the data.

Acknowledgements. We thank Marica Valentini for pointing us to the abundance measurements of the RAVE survey and for helpful discussion. S.U. acknowledges support from the Austrian Science Fund (FWF) under project P 22911N16 and from the Fund for Scientific Research of Flanders (FWO) under grant number G.0470.07. D.M.N. was primarily supported by the NSERC grant PGSD3-403304-2011, and partially supported by the NSF grant AST-1103471. T.L. acknowledges support from the FWF under projects P 23737-N16 and P 21988-N16. The Besançon model simulations were executed on computers from the Utinam Institute of the Université de Franche-Comté, supported by the Région de Franche-Comté and Institut des Sciences de l'Univers (INSU). We acknowledge the support of the French Agence Nationale de la Recherche under contract ANR-2010-BLAN-0508-01OTP. This publication makes use of data products from the Two Micron All Sky Survey, which is a joint project of the University of Massachusetts and the Infrared Processing and Analysis Center/California Institute of Technology, funded by the National Aeronautics and Space Administration and the National Science Foundation.

\section{References}

Aguerri, J. A. L., Balcells, M., \& Peletier, R. F. 2001, A\&A, 367, 428 Alves-Brito, A., Meléndez, J., Asplund, M., Ramírez, I., \& Yong, D. 2010, A\&A, 513, A35

Aringer, B., Girardi, L., Nowotny, W., et al. 2009, A\&A, 503, 913 Athanassoula, E. 2005, MNRAS, 358, 1477

Babusiaux, C., Gómez, A., Hill, V., et al. 2010, A\&A, 519, A77

Ballero, S. K., Matteucci, F., Origlia, L., \& Rich, R. M. 2007, A\&A, 467, 123

Bensby, T., Zenn, A. R., Oey, M. S., \& Feltzing, S. 2007, ApJ, 663, 13

Bensby, T., Feltzing, S., Johnson, J. A., et al. 2010a, A\&A, 512, A41

Bensby, T., Alves-Brito, A., Oey, M. S., Yong, D., \& Meléndez, J. 2010b, A\&A, 516, L13

Bensby, T., Adén, D., Meléndez, J., et al. 2011, A\&A, 533, A134

Bienaymé, O., Robin, A. C., \& Crézé, M. 1987, A\&A, 186, 359

Blanco, V. M., \& Terndrup, D. M. 1989, AJ, 98, 843

Boeche, C., Siebert, A., Williams, M., et al. 2011, AJ, 142, 193

Brown, J. M., Kilic, M., Brown, W. R., \& Kenyon, S. J. 2011, ApJ, 730, 67

Caffau, E., Ludwig, H.-G., Steffen, M., et al. 2008, A\&A, 488, 1031

Castellani, M., \& Castellani, V. 1993, ApJ, 407, 649

Chiappini, C., Górny, S. K., Stasińska, G., \& Barbuy, B. 2009, A\&A, 494, 591

Combes, F., \& Sanders, R. H. 1981, A\&A, 96, 164

Combes, F., Debbasch, F., Friedli, D., \& Pfenniger, D. 1990, A\&A, 233, 82

de Propris, R., Rich, R. M., Kunder, A., et al. 2011, ApJ, 732, L36

Durand, S., Acker, A., \& Zijlstra, A. 1998, A\&AS, 132, 13

Eggen, O. J., Lynden-Bell, D., \& Sandage, A. R. 1962, ApJ, 136, 748

Elmegreen, B. G., \& Elmegreen, D. M. 2005, ApJ, 627, 632

Elmegreen, B. G., Zhang, H.-X., \& Hunter, D. A. 2012, ApJ, 747, 105

Fluks, M. A., Plez, B., The, P. S., et al. 1994, A\&AS, 105, 311

Fuhrmann, K. 2011, MNRAS, 414, 2893

Fulbright, J. P., McWilliam, A., \& Rich, R. M. 2007, ApJ, 661, 1152

Fux, R. 1999, A\&A, 345, 787

Genzel, R., Newman, S., Jones, T., et al. 2011, ApJ, 733, 101

Girardi, L., Bressan, A., Bertelli, G., \& Chiosi, C. 2000, A\&AS, 141, 371

Girard, T. M., van Altena, W. F., Zacharias, N., et al. 2011, AJ, 142, 15

Girardi, L., Groenewegen, M. A. T., Hatziminaoglou, E., \& da Costa, L. 2005, A\&A, 436, 895

Gonzalez, O. A., Rejkuba, M., Zoccali, M., et al. 2011a, A\&A, 530, A54

Gonzalez, O. A., Rejkuba, M., Zoccali, M., Valenti, E., \& Minniti, D. 2011b, A\&A, 534, A3

Gomez, A. E., Grenier, S., Udry, S., et al. 1997, Proceedings of the ESA Symposium "Hipparcos - Venice '97", ESA SP-402, 621

Guzman-Ramirez, L., Zijlstra, A. A., NíChuimín, R., et al. 2011, MNRAS, 414, 1667

Haywood, M. 2008, MNRAS, 388, 1175

Hinkle, K., Wallace, L., Valenti, J., \& Harmer, D. 2000, Visible and Near Infrared Atlas of the Arcturus Spectrum 3727-9300 A, (San Francisco: ASP)

Hill, V., Lecureur, A., Gómez, A., et al. 2011, A\&A, 534, A80

Houdashelt, M. L., Bell, R. A., Sweigart, A. V., \& Wing, R. F. 2000, AJ, 119, 1424

Howard, C. D., Rich, R. M., Reitzel, D. B., et al. 2008, ApJ, 688, 1060

Howard, C. D., Rich, R. M., Clarkson, W., et al. 2009, ApJ, 702, L153

Inoue, S., \& Saitoh, T. R. 2012, MNRAS, 422, 1902

Ivezić, Z̆., Sesar, B., Jurić, M., et al. 2008, ApJ, 684, 287

Johnson, C. I., Rich, R. M., Fulbright, J. P., Valenti, E., \& McWilliam, A. 2011a, ApJ, 732, 108

Johnson, J. A., Clanton, C., Howard, A. W., et al. 2011b, ApJS, 197, 26

Jørgensen, U. G. 1997, in Molecules in Astrophysics: Probes and Processes, ed.

E. F. van Dishoeck (Kluwer), IAU Symp., 178, 441

Kormendy, J., \& Kennicutt, R. C. 2004, ARA\&A, 42, 603

Kunder, A., Koch, A., Rich, R. M., et al. 2012, AJ, 143, 57

Kupka, F., Piskunov, N., Ryabchikova, T. A., Stempels, H. C., \& Weiss, W. W. 1999, A\&AS, 138, 119

Lebzelter, T., Uttenthaler, S., Busso, M., Schultheis, M., \& Aringer, B. 2012, A\&A, 538, A36

Lejeune, Th., Cuisinier, F., \& Buser, R. 1997, A\&AS, 125, 229

Lejeune, Th., Cuisinier, F., \& Buser, R. 1998, A\&AS, 130, 65

Li, Z.-Y., \& Shen, J. 2012, ApJ, 757, L7

Marshall, D. J., Robin, A. C., Reylé, C., Schultheis, M., \& Picaud, S. 2006, A\&A, 453, 635

McWilliam, A., \& Zoccali, M. 2010, ApJ, 724, 1491

Meléndez, J., Asplund, M., Alves-Brito, A., et al. 2008, A\&A, 484, L21

Nataf, D. M., Udalski, A., Gould, A., Fouqué, P., \& Stanek, K. Z. 2010, ApJ, 721, L28

Nataf, D. M., Gould, A. P., Pinsonneault, M. H., \& Udalski, A. 2012, ApJ, submitted [arXiv: 1109.2118]

Ness, M., Freeman, K., Athanassoula, E., et al. 2012, ApJ, 756, 22

Ng, Y. K. 1997, A\&A, 328, 211 
S. Uttenthaler et al.: The structure and formation of the Galactic bulge

Noguchi, M. 1998, Nature, 392, 253

Noguchi, M. 1999, ApJ, 514, 77

Norman, C. A., Sellwood, J. A., \& Hasan, H. 1996, ApJ, 462, 114

Norris, J., Bessell, M. S., \& Pickles, A. J. 1985, ApJS, 58, 463

Ojha, D. K., Bienayme, O., Robin, A. C., Creze, M., \& Mohan, V. 1996, A\&A,

$$
311,456
$$

Perea-Calderón, J. V., García-Hernández, D. A., García-Lario, P., Szczerba, R. \& Bobrowsky, M. 2009, A\&A, 495, L5

Raha, N., Sellwood, J. A., James, R. A., \& Kahn, F. D. 1991, Nature, 352, 411

Reylé, C., \& Robin, A. C. 2001, A\&A, 373, 886

Reylé, C., Marshall, D. J., Robin, A. C., \& Schultheis, M. 2009, A\&A, 495, 819

Rich, R. M. 2011, Carnegie Obs. Astrophys. Ser., 5, 264

Rich, R. M., Origlia, L., \& Valenti, E. 2012, ApJ, 746, 59

Rich, R. M., Origlia, L., \& Valenti, E. 2007, ApJ, 665, L119

Robin, A. C., Reylé, C., Derrière, S., et al. 2003, A\&A, 409, 523

Robin, A. C., Marshall, D. J., Schultheis, M., \& Reylé, C. 2012, A\&A, 538, A106
Ryde, N., Gustafsson, B., Edvardsson, B., Meléndez, J., \& Alves-Brito, A. 2010 A\&A, 509, A20

Saito, R. K., Zoccali, M., McWilliam, A., et al. 2011, AJ, 142, 76

Schultheis, M. 1998, Ph.D. Thesis, University of Vienna, Austria

Schwenke, D. 1998, Faraday Discussions, 109, 321

Shen, J., Rich, R. M., Kormendy, J., et al. 2010, ApJ, 720, L72

Skrutskie, M. F., Cutri, R. M., Stiening, S., et al. 2006, AJ, 131, 1163

Thomas, D., Maraston, C., Bender, R., \& Mendes de Oliveira, C. 2005, ApJ, 621, 673

Twarog, B. A. 1980, ApJS, 44, 1

Uttenthaler, S., Hron, J., Lebzelter, T., et al. 2007, A\&A, 463, 251

Vanhollebeke, E., Groenewegen, M. A. T., \& Girardi, L. 2009, A\&A, 498, 95

Zhao, H. 1996, MNRAS, 283, 149

Zoccali, M., Renzini, A., Ortolani, S., et al. 2003, A\&A, 399, 931

Zoccali, M., Hill, V., Lecureur, A., et al. 2008, A\&A, 486, 177

Zwitter, T., Siebert, A., Munari, U., et al. 2008, AJ, 136, 421 